\title{
Challenges in Advancing the Field of Cancer Gene Therapy: An Overview of the Multi-Functional Nanocarriers
}

\author{
Azam Bolhassani and Tayebeh Saleh \\ Additional information is available at the end of the chapter \\ http://dx.doi.org/10.5772/54862
}

\section{Introduction}

Recent developments in molecular biology and cell biology have led to the discovery of novel genes and proteins having therapeutic potentials for various diseases including cancers. Based on these findings, novel categories of therapeutic biomacromolecules including genes, small interfering RNA (siRNAs), antisense oligonucleic acids, bioactive proteins and peptides have been developed. These macromolecules can be more advantageous than small-molecular-weight therapeutic agents in terms of their specificity and high potency to the target molecules [Nakase et al., 2010]. Gene therapy is the newest therapeutic strategy for treating human diseases. The basic idea of gene therapy is a gene or gene product that can be selectively delivered to a specific cell/tissue with minimal toxicity. This product can inhibit the expression of a specific defective gene or express a normal gene. Efficient and safe delivery is one of the key issues for the clinical application of nucleic acids as therapeutic agents [Du et al., 2010]. The goal of the Pharmaceutical Industry is to have a gene therapy medical product that can be delivered systemically. In vivo gene therapies have focused on viral vectors for gene delivery and have had marginal clinical successes. Major disadvantage of these delivery systems is the integration of some viral vectors into human chromosomes of normal tissue. There are four issues to be solved before cancer gene therapy will be successful: 1) Identification of key target genes critical for the disease pathology and progression; 2) Determination of the correct therapeutic gene to inhibit disease progression; 3) Optimal trans-gene expression for suppressing the target gene; and 4) Delivery of therapeutic product to the target tissue at an efficient dose [Scanlon, 2004]. 
Delivery is one of the most difficult challenges facing the gene therapy field. It is needed to use an efficient transfer system that can stabilize, transduce and express a transgene in the target tissue. Recently, non-viral technologies have been widely used as a significant alternative for gene delivery. The non-viral delivery systems have reduced adverse immune responses, are easier to manufacture and can be produced for the pharmaceutical industry in large quantities. The current progresses of gene therapy are further focused on synthesized nano-particle technologies. Some of these new chemical compositions are cationic molecules such as polymers, lipids and peptides [Scanlon, 2004; Gao et al., 2007].

However, for successful clinical trial, ideal non-viral vectors should be degradable into low molecular weight components, in response to biological stimuli, for lowered cytotoxicity and effective systemic clearance. They should also be efficient in overcoming extracellular and intracellular barriers, tissue/cell-targeted for specific accumulations and multi-functional for synergistic therapeutic and diagnostic outcomes. Recently, a broad range of different stimuliresponsive strategies (virus-mimicking gene delivery systems) has been employed to develop non-viral nucleic acid carriers that efficiently enhance multiple extracellular and intracellular gene delivery pathways by altering their physico-chemical properties in response to a variety of extra- and intra-cellular stimuli (e.g., $\mathrm{pH}$, redox potential, and enzyme), as well as external triggers (e.g., light) [Zhu and Torchilin, 2012].

Therefore, numerous challenges remain to be overcome before gene therapy becomes available as a safe and effective treatment option. For instance, the cationic molecules (e.g., polymers, lipids and peptides) have the potential to be systemically delivered but selectivity for the target tissue needs to be validated. The best method for delivering genes will depend on the type of tissue targeted [Scanlon, 2004]. Recently, the development of virus-mimicking, multi-functional gene delivery systems is considered to be a potent strategy in the future, in particular for intravenous administration. This chapter summarizes the challenges for cancer gene therapy as well as addressing the advances in the multi-functional nano-carriers as a potent non-viral delivery system.

\section{Gene therapy}

Medicine has a long history of treating patients with cell therapies (i.e., blood transfusions) and protein therapies (i.e., growth factors and cytokines). Gene therapies are the newest therapeutic strategy for treating human diseases especially cancer [Scanlon, 2004]. This technology has been used to develop new strategies for killing cells selectively or inhibiting their growth. The field of cancer gene therapy comprises a range of technologies from direct attack on tumor cells to inducing the immune response to tumor antigens [McCormick, 2001]. However, there are serious doubts about gene therapy; for example, short-lived nature of gene therapy, immune response to a foreign object, problems with viral vectors and insertional mutagenesis inducing a tumor [Korthof, 1999]. 


\subsection{History}

In 1972, Friedmann and Roblin published a paper in Science entitled as "Gene therapy for human genetic disease" [Friedmann and Roblin, 1972; Rogers, 1970]. The first approved gene therapy case in the United States took place in 1990, at the National Institute of Health. It was performed on a four year old girl with a genetic defect associated to an immune system deficiency. The effects were only temporary, but successful [Blaese et al., 1995]. In addition, sickle cell disease was successfully treated in mice [Fisher, 1995]. At the same time, the researchers were able to create tiny liposomes 25 nanometers $(\mathrm{nm})$ that can carry therapeutic DNA through pores in the nuclear membrane [www. newscientist.com, 2002]. In 1992, Claudio Bordignon performed the first procedure of gene therapy using hematopoietic stem cells as vectors to deliver genes aimed to correct hereditary diseases [Abbott, 1992]. In 2002, this work led to the publication of the first successful gene therapy treatment for adenosine deaminase-deficiency (SCID) [Cavazzana-Calvo et al., 2004]. In 2003, a Los Angeles research team inserted genes into the brain using liposomes coated in a polymer called polyethylene glycol (PEG). The transfer of genes into the brain is a significant achievement because viral vectors are too big to get across the blood-brain barrier. This method had potential for treating Parkinson's disease. At the same time, RNA interference or gene silencing was considered as a new way to treat Huntington's disease [www. newscientist.com, 2003]. In 2006, scientists at the National Institutes of Health have successfully treated metastatic melanoma in two patients using killer T cells genetically retargeted to attack the cancer cells. For the first time, this study demonstrated that gene therapy can be effective in treating cancer. In November 2006, Preston Nix reported on VRX496, a gene-based immunotherapy for the treatment of human immunodeficiency virus (HIV) that uses a lentiviral vector for delivery of an antisense gene against the HIV envelope [Levine et al., 2006; www.eurekalert.org, 2009].

Leber's congenital amaurosis is an inherited blinding disease caused by mutations in the RPE65 gene. In 2007-2008, the world's first gene therapy trial was announced for inherited retinal disease. They determined the safety of the sub-retinal delivery of recombinant adenoassociated virus (AAV) carrying RPE65 gene and found the positive results in vision and without the apparent side-effects [www. news.bbc.co.uk, 2007]. In 2009, the researchers were succeeded at arresting a fatal brain disease, adrenoleukodystrophy, using a vector derived from HIV to deliver the gene for the missing enzyme [Kaiser, 2009]. A paper published in 2010, deals with gene therapy for a form of achromatopsia in dogs. Achromatopsia, or complete color blindness, is presented as an ideal model to develop gene therapy directed to cone photoreceptors [Komáromy et al., 2010]. In 2011, a study carried out using genetically modified $\mathrm{T}$ cells to fight the chronic lymphocytic leukemia (CLL) disease. Moreover, Human HGF plasmid DNA therapy of cardiomyocytes was examined as a potential treatment for coronary artery disease as well as myocardial infarction [www. nature.com, 2011; Yang et al., 2008; Hahn et al., 2011]. However, most of the approved European and United States gene therapy protocols are for cancer $(\sim 66 \%)$, in contrast to monogenetic diseases $(\sim 11 \%)$ and cardiovascular diseases $(\sim 8 \%)$. The focus of cancer gene therapy has been on melanoma, prostate and ovarian cancer and leukemia [Scanlon, 2004]. 


\subsection{The challenges of gene therapy}

Recently, the use of gene therapy in medicine using plasmid DNA (pDNA), oligodeoxynucleotide $(\mathrm{ODN})$ or small interfering RNA (siRNA) represents a promising new approach for treating a variety of genetic and acquired diseases (e.g., cancer). To date, more than 1000 different gene-therapy clinical trials for the treatment of many different diseases are in progress worldwide, but, the success with gene therapy has been limited. This lack of success can be assigned to difficulties related to the effective delivery of nucleic acids into target cells [Mastrobattista et al., 2006]. Naked DNA is unable to efficiently cross cellular barriers by passive diffusion because of its large size, strong negative charge, hydrophilicity and susceptibility to nuclease attack. Hence, the major challenge for in vitro DNA delivery is designing suitable vectors which can protect DNA and efficiently deliver it to the targeted sites in the cell. Successful application of such therapeutic vector-DNA formulations in vivo requires them to be stable during circulation in blood, resistant against rapid metabolic clearance and efficiently targeted to the appropriate tissue/cell [Mann et al., 2008].

\section{Delivery systems in gene therapy}

\subsection{Viral delivery}

Delivery is one of the most difficult challenges facing the gene therapy field. An efficient transfer system has not yet been found to stabilize, transduce and express a transgene in the target tissue. Limitations of the present vector technologies have slowed the progress of clinical gene therapy [Scanlon, 2004]. Various viruses, such as influenza viruses and adenoviruses, can efficiently deliver genes into the nucleus via sophisticated mechanisms. Despite the potent immunogenicity of viral vectors, their developed cell entry mechanism and high transfection efficiency in both dividing and non-dividing cells is desirable [Wagner, 2011; Mastrobattista et al., 2006]. All the viral gene strategies used to date have significant delivery limitations. The best method for delivering genes may depend on the type of targeted tissue. There are some promising delivery technologies for viral therapies, including the use of replication competent viruses. Adenoviruses, herpes simplex virus and Newcastle disease virus have all been modified for replication competent properties in human tumor cells. This has been one of the most popular areas in gene therapy and offers promises for treating cancer, especially when combined with chemotherapy [Scanlon, 2004]. Recently, the viral vectors have been developed into gene vectors and have provided convincing successes in gene therapy. Viruses have developed mechanisms to survive in the extracellular environment, attach to cells, cross cellular membranes, steal intracellular transport systems and subsequently deliver their genomes into the appropriate sub-cellular compartment (e.g., cytosol or nucleus) [Wagner, 2011; Sasaki et al., 2008]. For example, influenza viruses infect cells in a multi-step process: a) the virus binds to a receptor on the cell surface mediated by hemagglutinin (HA) protein; $b$ ) the virus invades via receptor-mediated endocytosis; c) the internalized virus is trafficked to a late endosome; $d$ ) the acidic endosomal environment induces membrane fusion between the virus and endosome, which is brought about by a conformational change of HA and the ribonucleoprotein complex 
core is released into cytoplasmic space; e) the core is transferred to the nucleus and viral gene expression progresses. Due to this very efficient cell-entry mechanism, the transfection efficiencies of viral vectors remain uniqe [Sasaki et al., 2008].

However, there are several problems to overcome before this therapy can be successful in clinical settings such as a) Enhanced lytic properties of these viruses; b) Improved yields with better manufacturing procedures of production for clinical studies; c) Systemic delivery; d) limited DNA-carrying capacity; e) lack of target-cell specificity; f) immunogenicity and h) for some viral vectors, insertional mutagenesis [Scanlon, 2004; Mastrobattista et al., 2006].

\subsection{Non-viral delivery}

There are some non-viral technologies that offer several advantages over the viral methodologies. Non-viral delivery systems have reduced adverse immune responses (relatively safe), are easier to manufacture, can be produced for the pharmaceutical industry in large quantities and modified by the incorporation of ligands for targeting to specific cell types [Scanlon, 2004; Mastrobattista et al., 2006]. Chemically synthesized nanoparticles constitute a new technology and offer several new strategies for successful systemic gene therapy delivery. Synthetic gene-delivery systems consist of a self-assembling complex of DNA with positively charged molecules (for example, polymers, peptides, lipids or their combinations). These complexes are small in size $(40-150 \mathrm{~nm})$ and usually have a net positive surface charge, which enables adsorption-mediated cell binding and internalization [Mastrobattista et al., 2006]. Some of these new chemical compositions are polymers containing either DNA/stearyl polylysine-coated lipids or peptoids (DNA coated with glycine oligomers) or cationic molecules (DNA/combined with positively charged B-cyclodextrin/adamantane and PEG). These molecules have been shown to be effective in cancer-related angiogenesis. These new agents have the potential to be systemically delivered, but their selectivity for the target tissue needs to be validated [Scanlon, 2004]. However, the levels of gene expression and the transfection efficiency mediated by synthetic vectors are low compared to viral vectors [Mastrobattista et al., 2006].

\subsection{Cell delivery}

One of the opportunities for gene therapy is to combine therapeutic genes with a cell to overcome the delivery to target tissues. The advantages of cell delivery of therapeutic products include minimal immune response; tissue directed therapy; selectivity and improved potency of the product. However, there are several problems to be solved: a) Determining optimal transduction of cells; b) Gene-transformed cells will require a selective growth advantage over defective cells to re-populate the host; c) DNA repair genes action (minimized mutations in the gene-transformed cells); d) Genomic stability (for optimal gene expression); e) Determining cell type for therapy (e.g., embryonic stem cells or activated, differentiated cells); f) Incorporation of a safety mechanism (i.e. a suicide gene) to destroy the gene-transformed cells if a problem arises. In addition, other cell therapies are currently being developed using bacteria, such as modified Salmonella, for gene delivery in cancer patients. These modified bacterial cells are already in Phase I clinical studies [Scanlon, 2004]. 


\section{The challenges of nucleic acid delivery}

There are many biological barriers for efficient gene delivery that need to be overcome [Mastrobattista et al., 2006]. This inefficiency of gene delivery is primarily a result of the inability of these vectors to overcome the numerous barriers encountered between the site of administration and localization in the cell nucleus. This series of barriers to efficient non-viral gene delivery is thought to include: a) the physical and chemical stability of DNA and its delivery vehicle in the extracellular space, b) cellular uptake by endocytosis, c) escape from the endosomal compartments prior to trafficking to lysosomes, d) cytosolic transport and e) nuclear localization of the plasmid for transcription. In addition to these physical and chemical obstacles, biological barriers, such as immunogenic responses to the vector itself as well as immune stimulation by certain DNA sequences containing a central un-methylated CpG motif, are present. The studies have shown that it is possible to minimize biological barriers by optimizing the plasmid sequence, thus physical and chemical barriers appear to be the negative factors to successful non-viral gene delivery [Wiethoff and Middaugh, 2003]. These barriers are briefly described as following:

\subsection{Stability in extracellular compartments}

The stability of non-viral delivery systems in the extracellular milieu, such as intercellular or intravascular spaces, is related to the chemical stability of the DNA as well as the physical stability of the delivery system [Wiethoff and Middaugh, 2003]. In extracellular environment, the carrier is exposed to blood components such as nucleases. This can result in premature destabilization with simultaneous release and degradation of the plasmid DNA. The half-life of naked plasmid DNA in blood is on the order of minutes. Therefore, carrier-mediated protection during transport through the blood circulation is a prerequisite to make the DNA inaccessible to degradative enzymes [Mastrobattista et al., 2006]. Condensing the DNA with a variety of polycations or by complexing with polymers that bind to DNA protects it from degradation [Wiethoff and Middaugh, 2003].

\subsection{Cellular association of DNA}

Irrespective of the injection route, the gene carriers should be able to bind to cells for allowing cellular uptake [Mastrobattista et al., 2006]. Association of DNA with the cell surface is typically very low in the absence of any delivery agent as an immediate consequence of the relatively high negative charge density of both the DNA and the cell surface. Polycations have been shown to substantially increase the cellular association of DNA by neutralization of the DNA negative charge, with the charge ratio of the complex modulating the extent of this contact. The degree of cellular association has also been shown to be related to the colloidal stability of these delivery systems, with those that aggregate often manifesting a greater degree of cellular association in vitro. The association of non-viral gene delivery systems containing either cationic lipids or polymers is thought to be mediated by interactions with cell surface heparin sulfate proteoglycans (HSPGs). These proteoglycans are ubiquitous to the surface of all cells and are involved 
in a variety of cellular processes, including differentiation, adhesion and migration. More recently they have been found to mediate the binding and internalization of several viruses, including HIV-1, HSV-2, AAV-2, and adenovirus. The composition of heparin sulfate proteoglycans (HSPGs) includes a protein core with one or more attached glycosaminoglycans [Wiethoff and Middaugh, 2003].

In general, binding of nano-carrier to the cell surfaces is not, a problem if the gene carriers have a net positive surface charge, which readily induces adsorption onto negatively charged cell membranes. However, this form of binding is random and does not allow restricted delivery to target cells (e.g., tumor cells) [Mastrobattista et al., 2006]. Several additional strategies have been attempted to increase the specificity of DNA binding to cells and induce targeting to particular cell types [Wiethoff and Middaugh, 2003]. Active targeting can be achieved by the functionalization of NPs with ligands such as antibodies, peptides, nucleic acids (aptamers), carbohydrates and small molecules [Gu et al., 2007].

\subsection{Intracellular trafficking of non-viral gene delivery systems}

Since many chemotherapeutic drugs and particularly gene therapeutics, would benefit from intracellular targeting, nano-carrier systems can be designed for receptor-mediated cell uptake, intracellular drug protection and intracellular target delivery [van Vlerken et al., 2007]. Once inside the cell, several intracellular barriers need to be crossed before the foreign DNA can be transcribed and translated [Mastrobattista et al., 2006]. These barriers contain endosomal escape, cytosolic transport of DNA and nuclear localization of plasmid DNA that described briefly in below:

\subsubsection{Endosomal escape}

The endocytic pathway is one of the uptake mechanisms of cells. In general, non-viral nano vectors have been developed to mimic the receptor-mediated cell entry mechanism of viruses and the main mechanism of internalization was confirmed to be endocytosis. This pathway is composed of vesicles known as endosomes with an internal $\mathrm{pH}$ around 5 that mature in a unidirectional manner from early endosomes to late endosomes before fusing with intracellular organelles called lysosomes which contain certain digestive enzymes. Thus, particles entering the cells via the endocytic pathway become entrapped in endosomes and eventually end up in the lysosome, where active enzymatic degradation processes take place [Varkouhi et al., 2011]. The entrapment of internalized DNA carriers in endocytic compartments prevents further intracellular transport towards the nucleus and will often result in degradation of the carrier and its associated DNA in the endosomal/lysosomal compartments [Mastrobattista et al., 2006]. While many viruses have evolved quite efficient systems for endosomal release, the situation is different for non-viral vectors, where in many cases the lack of endosomal escape is a major obstacle for efficient biological delivery, implying that more efficient methods for endosomal release would lead to improvements in designing synthetic transfection systems. In contrast to synthetic vectors, viral vectors are known to be efficient both for in vitro and in vivo applications [Varkouhi et al., 2011]. 


\subsubsection{Cytosolic transport of DNA}

After endosomal escape, DNA must traverse the cytosol to access the nucleus. Those DNA carriers that manage to escape the endosomal compartments are then challenged by the complex environment of the cytosol, which contains many filamentous structures that hinder the free diffusion of large particles such as DNA carriers. Dissociation of the carrier at this stage might be required to allow further transport of the plasmid DNA molecules. However, several studies have found evidence that plasmid DNA is largely immobile in the cytosol and is rapidly degraded by cytosolic nucleases [Mastrobattista et al., 2006]. Diffusion of DNA in the cytoplasm has been found to be substantially less than that observed in dilute solution. For DNA $>2000$ base pairs in length, the diffusion co-efficient in the cytosol is $<1 \%$ of that in water, suggesting a substantial diffusional barrier. This decreased mobility has been ascribed to molecular crowding of the plasmid, but may also reflect an increased viscosity of the cytoplasm. As expected, the diffusion coefficient of DNA in the cytoplasm is inversely related to the size of the plasmid, suggesting smaller plasmids may be more desirable. No evidence for active transport of DNA in the cytoplasm has been reported. In addition to the considerable diffusional barrier for DNA in the cytosol, the presence of calciumsensitive cytosolic nucleases pose a significant metabolic barrier. Micro-injection of DNA into the cytoplasm results in significant degradation of the DNA with a half-life of 50-90 min [Wiethoff and Middaugh, 2003].

\subsubsection{Nuclear localization of plasmid DNA}

Ultimately, delivery of DNA to the nucleus must occur for transcription of the transgene to take place. The mechanism of DNA nuclear translocation and whether the DNA is still associated with the delivery system are not fully understood but appear to depend on the type of delivery vehicle employed. At least three possible routes exist for DNA transport to the nucleus. The DNA can pass into the nucleus through nuclear pores, it can become physically associated with chromatin during mitosis when the nuclear envelope breaks down or it could traverse the nuclear envelope. Of these three possibilities, the latter seems impossible and without experimental support. Nuclear pores are embedded in the nuclear envelope at fairly high surface densities (3000-4000/nucleus) and exist in at least two conformational states. The closed state permits the passive diffusion of molecules of $<9 \mathrm{~nm}$ in diameter, whereas the open state facilitates transport of particles $<26 \mathrm{~nm}$. This latter state could certainly assist the "threading" of supercoiled plasmid through the nuclear pore but not passage of typical nonviral gene delivery complexes [Wiethoff and Middaugh, 2003]. Small molecules ( $<40 \mathrm{kDa})$ can diffuse freely through the pores of the nuclear pore complexes (NPC), whereas larger molecules and particles (up to $40 \mathrm{~nm}$ in size) can only be imported through the NPC by an active transport mechanism [Mastrobattista et al., 2006]. In a few cases, collapsed particles of $<30 \mathrm{~nm}$ have been produced, which could presumably enter the nucleus by this mechanism. The second and perhaps quite widespread mechanism by which DNA is thought to gain access to the nucleus is by association with nuclear material on breakdown of the nuclear envelope during mitosis [Wiethoff and Middaugh, 2003]. In this case, the nuclear barrier breaks down, 
which explains the increased levels of transfection in dividing cells as compared to their growth-arrested counterparts. However, as most cells do not divide or divide only slowly, an active transport mechanism is needed to carry the DNA from the cytosol into the nucleus [Mastrobattista et al., 2006].

\subsection{Toxicity of non-viral delivery system}

An important obstacle to effective non-viral gene delivery is the cytotoxicity of the delivery vectors [Wiethoff and Middaugh, 2003]. Inside the nucleus, the transgene encoded on the plasmid vector should be expressed to establish therapeutic levels of recombinant proteins within the affected cell. This requires gene transcription regulatory elements such as promoters and enhancers, to drive the expression of the transgene in mammalian cells. Viral promoters are often used because of their strong transcriptional activation. However, their constitutive nature does not allow control over the level of transgene expression. For the expression of proteins with a narrow therapeutic window, tight control over the level of transgene expression is essential. In addition, the introduction of foreign DNA into mammalian cells can induce a profound immune response, presumably triggered by differences in the degree of methylation of the foreign DNA compared with the mammalian genome [Mastrobattista et al., 2006]. Induction of innate immune responses by un-methylated CpG sequences in plasmid DNA is perhaps a more serious concern because these species have been demonstrated to greatly reduce the efficiency of non-viral gene delivery. This immunotoxicity is thought to be related to the efficient transfection of immune cells because polycation/DNA complexes evoke a considerably greater immune response than DNA or cationic vector alone. Initial observations suggest that delivery systems that are phagocytosed, due to their large size, may promote a greater immune response. Removal of $\mathrm{CpG}$ motifs from DNA containing the transgene has proven a successful means of improving gene expression. The methods involved in producing vectors without these motifs are not insignificant, and currently present a major limitation to their widespread usage. It has therefore been proposed that avoidance of transfection of immune cells either by specific targeting to particular cell types or by manipulation of the physicochemical properties of the delivery systems is necessary for significant improvements in non-viral gene delivery [Wiethoff and Middaugh, 2003].

\section{Multi-functional nanocarriers}

Currently used pharmaceutical nanocarriers, such as liposomes, micelles, nanoemulsions, polymeric nano-particles and many others demonstrate a broad variety of useful properties including: a) longevity in the blood allowing for their accumulation in pathological areas; b) specific targeting to certain disease sites due to various targeting ligands attached to the surface of the nanocarriers; c) enhanced intracellular penetration with the help of surface-attached cellpenetrating molecules; d) contrast properties due to the carrier loading with various contrast materials allowing for direct carrier visualization in vivo; e) stimuli-sensitivity allowing for drug release from the carriers under certain physiological conditions and etc. Some of those pharmaceutical carriers have already made their way into clinic, while others are still under 
preclinical development. However, the combination of pharmaceutical nanocarriers with several mentioned abilities, are rare. For example, long-circulating immunoliposomes capable of prolonged residence in the blood and specific target recognition represent one of few examples of this kind. At the same time, the engineering of multi-functional pharmaceutical nanocarriers combinig several useful properties in one particle can significantly enhance the efficacy of many therapeutic and diagnostic protocols [Torchilin, 2006].

The use of cationic lipids and cationic polymers as transfection vectors for efficient intracellular delivery of DNA was suggested in 1987. Complexes between cationic lipids (such as Lipofectin $\AA$, an equimolar mixture of $\mathrm{N}-[1-(2,3-$ dioleyloxy) propyl]-N,N,N-trimethylammonium chloride-DOTMA and dioleoyl phosphatidylethanolamine -DOPE) and DNA (lipoplexes) and complexes between cationic polymers, such as PEI and DNA (polyplexes) are formed because of strong electrostatic interactions between the positively charged carrier and negatively charged DNA. A slight net positive charge of formed lipoplexes and polyplexes is believed to facilitate their interaction with negatively charged cells and improve transfection efficiency. Endocytosis (including the receptor mediated endocytosis) was repeatedly confirmed as the main mechanism of lipoplex/polyplex internalization by cells [Torchilin, 2006]. Of special importance is the fact that despite of endocytosis-mediated uptake lipolexes and polyplexes, DNA does not end in lysosomes but releases in the cytoplasm due to the destabilization of the endosomal membrane provoked by lipid or polymeric component of the complexes. In particular, lipoplexes fuse with the endosomal membrane when they contain a fusogenic lipid, dioleylphosphatidylethanolamine (DOPE), which easily undergoes the transition from bilayer to hexagonal phase facilitating the fusion. In case of polyplexes, which cannot directly destabilize the endosomal membrane, the mechanism of DNA escape from endosomes, is associated with the ability of polymers, strongly protonate under the acidic $\mathrm{pH}$ inside endosome and create a charge gradient ultimately provoking a water influx and endosomal swelling and disintegration. In both cases, DNA-containing complexes when released into the cytosol, dissociate allowing for nuclear entry of free DNA. Nuclear translocation of the plasmid DNA is relatively inefficient because of the barrier function of the nuclear membrane and small size of nuclear pores $(25 \mathrm{~nm})$, as well as DNA degrades fast under the action of cytoplasmic nucleases. It was estimated that only $0.1 \%$ of plasmids undergo nuclear translocation from the cytosol. The attachment of nuclear localization sequences to plasmid DNA may significantly enhance its nuclear translocation and transfection efficiency. New approaches in using multifunctional carriers for DNA delivery include the application of bimetallic nano-rods that can simultaneously bind compacted DNA plasmid and targeting ligands in a spatially defined manner [Torchilin, 2006].

DNA-lipid amphiphiles self-assemble into novel "DNAsomes"-liposome-like core-shell structures with subunits composed of branched DNA-lipid hybrid molecules. These DNAsomes can be precisely modified over a wide range in terms of both size and surface charge. More importantly, DNAsome is a natural carrier of small interfering RNA (siRNA) due to DNA-RNA base-pairing, enabling efficient co-delivery of drugs and siRNA. The DNAsome represents a universal multi-functional drug vector for simultaneous delivery of drugs, tracer dyes, or antibodies, along with genes, siRNA or antisense nucleic acids [Roh et al., 2011]. 


\section{Different types of nanocarrier systems}

Over the last eight years, a laboratory at Northeastern University has developed an array of multi-functional nanocarriers for the delivery of genes, drugs and imaging modalities. These flexible platforms consist of polymeric and lipid systems that combine different modalities and stimuli-responsive release properties [Jabr-Milane et al., 2008].

The diagnostic and/or therapeutic objectives of a multi-functional nanocarrier system determine the design of the formulation. A review of the literature shows that there are many different types of nanocarrier formulations for the diagnosis, imaging and treatment of a wide spectrum of diseases. These multi-functional carriers share three main design components: platform (core) material, encapsulated payload/biologically active agents and targeting/surface properties. Nanocarrier platforms can be categorized as organic-based, inorganic-based or a hybrid combination. Organic nano-platforms include polymeric nanocarriers, lipid-based nanocarriers (e.g., liposomes and nanoemulsions), dendrimers and carbon-based nanocarriers (e.g., fullerenes and carbon nanotubes) [Jabr-Milane et al., 2008]. Inorganic nano-platforms include metallic nanostructures, silica nanoparticles and quantum dots. An example of a hybrid platform is colloidal gold encapsulated in liposomes or superparamagnetic iron oxide particles encapsulated in polymeric nanoparticles. Selection of the core material is highly dependent on the properties of the biologically active agents. Inherent and dynamic properties of the agents such as therapeutic index, lipophilicity, charge and size should be considered. When combining therapeutic agents with each other, with an imaging/diagnostic modality, or with energy delivery, the interaction of system components (i.e., synergy, quenching, enhanced toxicity) and release kinetics should also be considered. Surface properties are the third design component of multi-functional nanocarriers. A common surface modification technique that decreases reticuloendothelial system (RES) clearance is the physical or covalent attachment of PEG chains to the nanocarrier platform. Since tumor microvasculature is known to be highly fenestrated, colloidal particles can accumulate by the enhanced permeability and retention (EPR) effect. PEG surface modification increases circulatory residence time, which increases the probability of accumulation at the target. Block co-polymers of poly (ethylene oxide) (PEO) and poly (propylene oxide) (PPO) (e.g., Pluronics $\left.{ }^{\circledR}\right)$ have also been used as surface conjugates to enhance circulation and achieve passive targeted delivery [Jabr-Milane et al., 2008].

Recently, nanoparticulate systems have been also developed for therapeutic gene delivery. Some researchers have developed glutathione-responsive nanoparticles for the delivery of plasmid DNA. The nanocarrier platform consisted of thiolated gelatin which was synthesized using 2-iminothiolane to covalently modify type B gelatin ( $\mathrm{pI} \sim 4.5)$. The release profiles of cross-linked and non-cross-linked thiolated gelatin nanoparticles and gelatin nanoparticles (control) loaded with fluorescein isothiocyanate-conjugated dextran (FITCdextran) were assessed in the presence of different glutathione concentrations (from 0.1 $\mathrm{mM}$ to $5 \mathrm{mM}$ ). A higher percentage of FITC-dextran was released from non-crosslinked nanoparticles compared to the cross-linked nanoparticles. The rate of FITC-dextran release from thiolated gelatin nanoparticles enhanced with increasing concentrations of gluta- 
thione. Glutathione in the media enhanced the release of FITC-dextran from thiolated gelatin nanoparticles by about $40 \%$, while only a $20 \%$ enhancement was seen with gelatin nanoparticles [Jabr-Milane et al., 2008]. After establishing the rapid, stimuli-responsive release profile of these particles, the thiolated gelatin nanoparticles were loaded with plasmid DNA expressing green fluorescent protein (GFP). Upon incubation of the formulations with murine fibroblast cells, fluorescence imaging revealed transfection and protein expression after $6 \mathrm{~h}$ continuing as long as $96 \mathrm{~h}$. Flow cytometry indicated that the cross-linked thiolated gelatin nanoparticles had the highest transfection efficiency. These nanocarriers are capable of rapid DNA delivery in response to intracellular glutathione. This nanocarrier platform was further modified to develop an anti-angiogenic gene therapy for the treatment of cancer. This platform consisted of PEG-modified thiolated gelatin nanoparticles loaded with plasmid DNA encoding the soluble form of the extracellular domain of vascular endothelial growth factor (VEGF) receptor-1 (sFlt-1). VEGF receptor over-expression in cancer is associated with neo-vascularization; sFlt-1 was selected as it blocks the VEGF receptor and the associated signal cascade [Jabr-Milane et al., 2008]. The PEG modified thiolated gelatin nanoparticles showed superior in vitro transfection in human breast adenocarcinoma cells when compared to plain gelatin nanoparticles, PEG-modified gelatin nanoparticles, thiolated gelatin nanoparticles, Lipofectin-plasmid DNA complexes and naked plasmid. In vivo evaluation of the formulation in $\mathrm{nu} / \mathrm{nu}$ mice bearing orthotopic human breast adenocarcinoma (MDA-MB-435) xenografts established transfection and expression of sFlt-1 as assessed by ELISA, western blot analysis, tumor volume, microvessel density and immunostaining. PEG-modified thiolated gelatin nanoparticles were effective in transfection with sFlt-1 expressing plasmid DNA in vivo and showed significant suppression of tumor growth in MDAMB-435 tumor-bearing mice. The expressed sFlt- 1 was able to suppress angiogenesis [Jabr-Milane et al., 2008]. As such, PEG-modified thiolated gelatin nanoparticles are a viable platform for the delivery of therapeutic DNA to tumor mass. In addition, a system was developed for oral gene delivery. The nanoparticles-in-microsphere oral system (NiMOS) consists of type B gelatin nanoparticles encapsulated in Poly-e-caprolactone (PCL) microspheres. Based on the successful transfection results with sFlt-1, type B gelatin nanoparticles were selected to encapsulate and deliver DNA, while PCL was selected to protect the nanoparticles from degradation in the stomach and deliver the particles to the intestine, where PCL is degraded by lipases. To evaluate the biodistribution of NiMOS, the researchers encapsulated 111In-radiolabeled gelatin nanoparticles in PCL microspheres, orally administered the formulation to fasted Winstar rats, harvested the tissues at different time points and compared the results to 111In-radiolabeled gelatin nanoparticles. The gelatin nanoparticles showed immediate and high accumulation in the large intestine whereas NiMOS accumulation was initially high in the stomach (after $1 \mathrm{~h}$ ) [Jabr-Milane et al., 2008]. It then transferred predominately to the large intestine after $2 \mathrm{~h}$. To explore the effect of this biodistribution profile on transfection, the formulations were loaded with reporter plasmids expressing $\beta$-galactosidase (CMV- $\beta$ gal) or expressing GFP. DNA loaded NiMOS were orally administered to fasted Winstar rats at an oral dose of $100 \mu \mathrm{g}$ plasmid DNA [Jabr-Milane et al., 2008]. 
Five days after administration, the rats were sacrificed and the GI tract was harvested for analysis. The results were compared to unloaded NiMOS formulations, naked plasmid and loaded gelatin nanoparticles. Transgene expression was evident in the small and large intestines with both reporter plasmids although GFP expression was more prominent with the loaded NiMOS formulation relative to the controls. Similar results were obtained in studies with Balb/c mice. Clearly, NiMOS is a promising system for targeted delivery of therapeutic DNA to the GI tract [Jabr-Milane et al., 2008].

\section{Design of multi-functional nanocarrier system}

An artificial gene-delivery vector, with a multi-component architecture is able to overcoming all the barriers, in which each component performs a different task in a planned fashion. The design of multi-functional nano-carrier is a multidisciplinary task that requires a profound understanding of the physico-chemical mechanisms that drive the assembly of such nanoparticles. One among many prerequisites for a successful carrier system for nucleic acids is high stability in the extracellular environment. In addition, the biological processes that elicit the cellular uptake and intracellular processing accompanied by an efficient release of the cargo in the intracellular compartment of these gene delivery systems have to be well understood. A promising strategy to create such an interactive delivery system is to exploit the various biological stimuli. With greater understanding of physiological differences between normal and disease tissues and advances in material design, there is an opportunity to develop nanocarrier systems for target-specific drug and gene delivery that will respond to the local stimuli [Qiao et al., 2010; Mastrobattista et al., 2006]. At present, many examples of versatile, selfassembling nano-particles for the delivery of DNA can be found in literature and this number is continuously growing. In this section, emphasis is placed on the functional components that are needed for effective gene delivery and also the biological stimuli such as $\mathrm{pH}$ and redox potential for the synthesis of multi-functional intelligent delivery systems. Briefly, Table 1 indicates these functional carriers.

\subsection{Bio-compatible, bio-degradable nano-carriers}

The artificial virus should preferably be constructed from materials that are biocompatible and biodegradable to prevent carrier-induced toxicities and the accumulation of carrier components in the body. In general, lipids are well tolerated. Synthetic polymers, on the other hand, have shown to induce some cytotoxicity in vitro and in vivo. It is difficult to predict, however, which polymer will be cytotoxic and which not on the basis of the structure of the cationic polymer. In general, low-molecular-mass cationic polymers are less toxic than high-molecularmass polymers. Peptides derived from L-amino acids are inherently biodegradable. However, when proteins or peptides contain large numbers of positively charged or exposed hydrophobic amino acids, they can destabilize biological membranes and thereby cause cytotoxicity [Mastrobattista et al., 2006]. 


\begin{tabular}{lll}
\hline Function & Examples & Reference \\
\hline Bio-compatible, biodegradable & Synthetic polymers: & van Vlerken et al., 2007; Mastrobattista \\
nanocarrier & Poly (D, L-lactide-coglycolide), Poly & et al., 2006 \\
& (L-lactic acid), \\
& Poly (epiloncaprolactone), \\
& Poly (alkylcyanoacrylates) \\
& Natural polymers: \\
& Gelatin, Chitosan, Hyaluronic acid
\end{tabular}

\begin{tabular}{|c|c|c|}
\hline $\begin{array}{l}\text { Packaging nucleic acids into } \\
\text { compact nanoparticles }\end{array}$ & $\begin{array}{l}\text { Polyethyleimine (PEI), } \\
\text { Polylysine (pLys) }\end{array}$ & Wagner, 2011; Walker et al., 2005 \\
\hline Long-circulation of nanocarrier & $\begin{array}{l}\text { Polysaccharides, Polyacrylamide, } \\
\text { Polyvinylalcohol, } \\
\text { PEG [PEGylated polyplexes] }\end{array}$ & $\begin{array}{l}\text { van Vlerken et al., 2007; Mastrobattista } \\
\text { et al., } 2006\end{array}$ \\
\hline $\begin{array}{l}\text { Targeting molecules for the } \\
\text { development of targeted NPs }\end{array}$ & $\begin{array}{l}\text { Monoclonal antibody, Affibody, } \\
\text { Aptamer, Oligopeptides, Growth } \\
\text { factor, Vitamin }\end{array}$ & $\begin{array}{l}\text { Wang et al., 2011; Wagner, 2011; } \\
\text { Mastrobattista et al., 2006; Gu et al., } \\
\text { 2007; van Vlerken et al., 2007; Peer et } \\
\text { al., 2007; Alexis et al., 2008; Farokhzad } \\
\text { et al., 2006; Zhang et al., 2001; } \\
\text { Askoxylakis et al., 2005; Soudy et al., } \\
\text { 2011; Kim et al., 2006; Ogris et al., } \\
2003\end{array}$ \\
\hline
\end{tabular}

\begin{tabular}{|c|c|c|}
\hline Intracellular delivery & $\begin{array}{l}\text { Escape from the endosomal } \\
\text { compartment: } \\
\text { Influenza HA2 peptide, } \\
\text { melittin, } \\
\text { T-domain of the diphtheria toxin, } \\
\text { GALA peptide, } \\
\text { pH-sensitive polymers: PEI } \\
\text { Cytosolic un-packaging: Reduction- } \\
\text { sensitive polyplexes } \\
\text { Nuclear Import: } \\
\text { SV40 from the large tumor antigen } \\
\text { Simian virus } 40 \text {, } \\
\text { M9 from nuclear ribonucleoprotein }\end{array}$ & $\begin{array}{l}\text { Eliyahu et al., 2005; Shim and Kwon, } \\
\text { 2012; Vercauteren et al., 2012; Du et } \\
\text { al., 2010; Schaffer et al., 2000; } \\
\text { Bauhuber et al., } 2009\end{array}$ \\
\hline
\end{tabular}

Table 1. The use of different formulations with specific functions for designing of multi-functional nanocarrier systems 
Polymeric nano-particles offer significant advantages over other nano-carrier platforms primarily since a remarkable flexibility in polymer matrices allows for tailoring of the nanoparticle properties to meet the specific planed need. Other advantages of polymeric nanoparticles include ease of production, ease of surface modification, encapsulation efficiency of the payload, payload protection, large area-to-volume, slow or fast polymer degradation and stimuli-responsive polymer erosion for temporal control over the release of drugs and feasibility of scale-up and manufacturing. Some examples of the most commonly used polymers for nano-carriers include the synthetic polymers such as poly (D, L-lactide-coglycolide) (PLGA), poly (L-lactic acid) (PLL), poly (epsiloncaprolactone) (PCL), poly (alkylcyanoacrylates) and natural polymers such as gelatin, chitosan and hyaluronic acid [van Vlerken et al., 2007; Mastrobattista et al., 2006].

\subsection{Packaging nucleic acids into compact nano-particles}

A prerequisite for every systemic nucleic-acid delivery system is stability in the blood stream prior to reaching its target cell. Therefore, the carrier must prevent the premature release of its load. Among the different delivery systems, nano-sized drug carriers are receiving considerable attention. Through precise selection of candidate therapeutics and appropriate functionalization of the nano-carrier systems, it is possible to develop fairly sophisticated multifunctional systems that can provide optimized anticancer therapy, a function that imparts particular use in intracellular delivery and sub-cellular localization of drugs. Reversible nucleic acid condensation by cationic proteins is a common natural process, e.g., in packaging of whole mammalian genomes into chromatin, or RNA into organelles. Compaction is also a key function of viral cores for protection against the degradative environment during infection. Nucleic acids as macromolecules are subjected to a variety of environmental factors such as $\mathrm{pH}$ or enzymes (e.g., nucleases) that can degrade or destroy them. Complexation of nucleic acids by cationic polymers or lipids is a widely used method to reduce their sizes and prevent their destruction by nucleases.

Reversibility is important; the delivered nucleic acid has to be accessible for subsequent transcription [Wagner, 2011]. Polyplexes are non-viral vectors consisting of DNA and polycations have shown potential in systemic targeted gene delivery in various animal models, but suffer from far lower gene transfer efficiency than viral vectors. To improve efficiency, researchers are trying to engineer synthetic vectors with virus-like qualities. For successful condensation of DNA into virus size particles, an excess of polycations is necessary, which results in a positive surface charge. The positive surface charge on particles has been shown to be generally advantageous for cell uptake [Walker et al., 2005]. Polyionic interactions, hydrogen bonding and hydrophobic interactions control the condensation of nucleic acids. In electrostatic complexes of plasmid DNA (pDNA) with polycations such as polylysine (pLys) or polyethylenimine (PEI), neutralization of approximately 10,000 negative phosphate charges of one pDNA molecule by approximately 100 polycation molecules results in compaction into "polyplexes" with sizes of 20 to $>100 \mathrm{~nm}$ (depending on aggregation events). The polymer/pDNA core may be regarded as the engine of the delivery vehicle; for efficient and specific delivery, like in natural viruses, additional domains for cell entry and endosomal escape are required [Fig.1A \& B; Wagner, 2011]. 


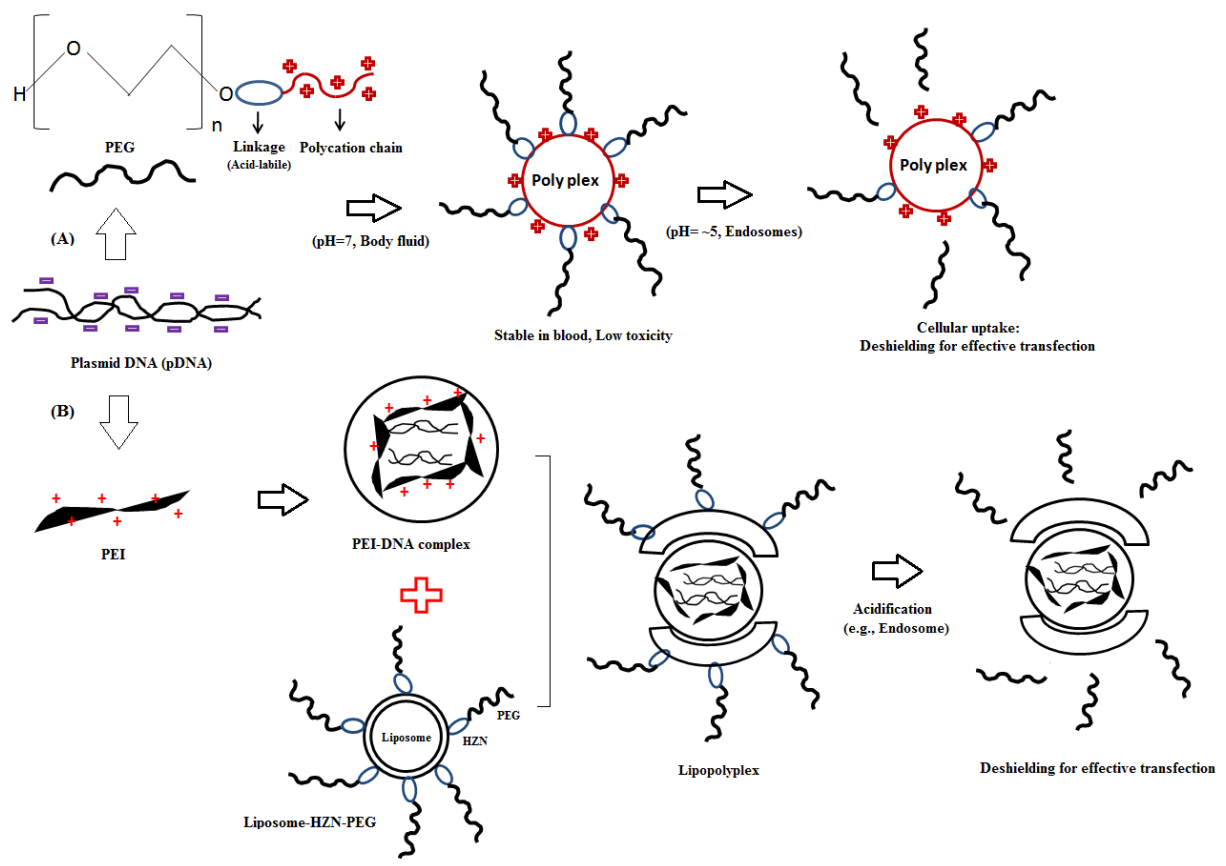

Figure 1. A) Schematic model of the pH-sensitive polyplexes: Covalent attachment of active targeting ligands e.g., peptides, proteins, aptamers and small molecules to PEG; Formation of the polycation- pDNA polyplex through charge-charge interaction; Complex shielded at physiological pH and deshielded at acidic pH. B) pH-reversible pEGylated lipopolyplexes: Formation of complex by mixing DNA and PEI (polyplex); Incubation of the cationic polyplexes with liposomes (liposome-Pyridylhydrazone-PEG); Shielding in extracellular compartments and deshielding in acidic medium (e.g., endosomes).

\subsection{Long-circulation of nanocarrier}

Both naked DNA and lipoplexes have showed rapid hepatic clearance during systemic administration. The liver elimination of lipoplexes was due to phagocytosis by Kupffer cells. Absence of any hydrophilic surface group on the particles, may lead to their interaction with plasma proteins, opsonization and removal from the circulation. The mononuclear phagocytic system (MPS) plays a key role in systemic removal of hydrophobic particles. In addition to biodegradability and biocompatibility, the non-viral carrier should be 'invisible' to the innate and acquired immune system of the patient in order to prevent unwanted immune reactions against the carrier and, consequently, rapid clearance of carriers from the blood circulation after intravenous administration. This can be achieved by adding a hydrophilic coat around the carrier. The coat can consist of a lipid bilayer or hydrophilic polymers grafted onto the surface of the carriers [Mastrobattista et al., 2006]. Among several strategies to impart particles with stealth-shielding, including surface modification with polysaccharides, polyacrylamide, and polyvinyl alcohol, surface modification with PEG and PEG co-polymers proved to be most 
effective, fueling its wide-spread use. PEG has a general structure of $\mathrm{HO}-\left(\mathrm{CH}_{2} \mathrm{CH}_{2} \mathrm{O}\right)_{\mathrm{n}}-$ $\mathrm{CH}_{2} \mathrm{CH}_{2}-\mathrm{OH}$, including a polyether backbone that is chemically inert, with terminal hydroxyl groups that can be activated for conjugation to different types of polymers and drugs. PEG offers the advantage that it is non-toxic and non-immunogenic, leading to approval by the United States Food and Drug Administration (FDA) for internal use in humans and inclusion in the list of inactive ingredients for oral and parenteral applications [Fig. 1 A \& B; van Vlerken et al., 2007]. The protective (stealth) action of PEG is mainly due to the formation of a dense, hydrophilic cloud of long flexible chains on the surface of the colloidal particle that reduces the hydrophobic interactions with the RES. The chemically anchored PEG chains can undergo spatial conformations, thus preventing the opsonization of particles by the macrophages of the RES, which leads to preferential accumulation in the liver and spleen. PEG surface modification, therefore, enhances the circulation time of molecules and colloidal particles in the blood. The mechanism of steric hindrance by the PEG modified surface has been thoroughly examined. The water molecules form a structured shell through hydrogen bonding to the ether oxygen molecules of PEG. The tightly bound water forms a hydrated film around the particle and prevents the protein interactions. In addition, PEG surface modification may also increase the hydrodynamic size of the particle decreasing its clearance, a process that is dependent on the molecular size as well as particle volume. Ultimately, this helps in greatly increasing circulation half-life of the particles [van Vlerken et al., 2007]. In cancer therapy, PEGylated polyplexes with elongated plasma circulation may take advantage of the "enhanced permeability and retention" (EPR) effect. Long-term circulating nano-particles can extravasate and passively accumulate at tumor sites due to the leakiness of tumor vessels and ineffective lymphatic efflux ("passive tumor targeting") [Wagner, 2011].

\subsection{Targeting molecules for the development of targeted NPs}

Appropriate packaging of nucleic acids and the use of PEG as a shield can help the complex survive within the circulation without being degraded or taken up by the mononuclear phagocyte system MPS. However, the next challenge for a PEGylated gene complex is to specifically target to cells or tissue of interest. By taking advantage of increased expression levels of receptors or antigens in diseased conditions, such as cancer, gene complexes can be targeted using specific ligands, such as antibodies, peptides, proteins, small molecules and RNA aptamer that recognize and bind to the cells of interest, resulting in high transfection efficiency [Wang et al., 2011].

Viruses are optimized for surviving in the relevant body fluids. Their surface is decorated with ligands for attachment to their target cell surface receptors. Often they use more than one receptor type for intracellular uptake into host cells. In design of synthetic nano-particles, such multivalent recognition and cell uptake mechanisms for nucleic acid delivery can be utilized [Wagner, 2011]. A simultaneous effect of the surface modification of gene carriers are that the positive surface charges are shielded, which significantly reduces the non-specific adsorption onto cell membranes. This enables targeting of the gene carriers towards specific cell types by conjugating ligands to the hydrophilic coat around the gene carrier that specifically bind internalizing cell-surface receptors. In this way, delivery of the transgene and subsequent 
expression can be restricted to target cells. Several different types of targeting ligands have been used for this purpose, including peptides, antibodies and vitamins. If targeting ligands are directed towards internalizing receptors, receptor binding will lead to receptor-mediated endocytosis of the targeted gene carriers, as they are small enough $(<200-300 \mathrm{~nm})$. This route of uptake is to be preferred as it ensures intracellular accumulation of gene carriers in a receptor-specific way [Mastrobattista et al., 2006]. While it has been demonstrated that PEG surface modification of nano-carriers causes a greater accumulation of drug at the tumor-site by passive targeting, active targeting of the carrier can help in selection of the target cell-type within the tumor site and internalization of the nano-particles to a greater extent inside the target cells. Active targeting can be achieved by the functionalization of NPs with ligands such as proteins (mainly antibodies and their fragments), nucleic acids (aptamers), or other receptor ligands (peptides, carbohydrates and vitamins) [Gu et al., 2007]. Regardless of the targeting moiety, the principle outcome is essentially the same, mainly improved tumor cell recognition, improved tumor cell uptake, and reduced recognition at non-specific sites. PEG surface modification provides an advantage whereby the terminal groups of PEG can be functionalized to reactive groups for covalent coupling. Most commonly, PEG is functionalized to reactive carboxylic acids, amine, or sulfhydryl groups, allowing for efficient covalent attachment of the wide variety of targeting ligands by amide bonding or disulfide bridge formation [van Vlerken et al., 2007]. In this section, we describe some classes of targeting molecules.

\subsubsection{Monoclonal antibodies}

Monoclonal antibodies $(\mathrm{mAb})$ are the first and are still the preferred class of targeting molecules. The mAb Rituximab was approved by the FDA for treating B-cell lymphoma in 1997. Another successful therapeutic mAb is Trastuzumab (Herceptin), an anti-HER2 mAb which binds to ErbB2 receptors and was approved by the FDA for treating breast cancer in 1998 . Cetuximab, which binds to epidermal growth factor receptors (EGFR), was approved for treating colorectal cancer in 2004 and head/neck cancer in 2006. Bevacizumab, a tumor angiogenesis inhibitor that binds to vascular endothelial growth factor (VEGF), was approved for treating colorectal cancer in 2004. Trastuzumab and rituximab have been conjugated to poly (lactic acid) (PLA) NPs resulting in conjugates that exhibit a six-fold increase in the rate of particle uptake compared with similar particles lacking the mAb targeting. Today, over 200 delivery systems based on antibodies or their fragments are in preclinical and clinical trials. Antibodies may be used in their native state or as fragments for targeting [Imai and Takaoka, 2006; Peer et al., 2007].

\subsubsection{Affibodies as targeting ligands}

In recent times, a novel class of small molecules called "affibodies," which can be considered antibody mimics, have been examined for targeting. Affibodies are a class of polypeptide ligands that are potential candidates for tissue-specific targeting of drug-encapsulated controlled release polymeric nanoparticles. Affibody molecules are relatively small proteins (6-8 $\mathrm{kDa})$ that offer the advantage of being extremely stable, highly soluble, and readily expressed in bacterial systems or produced by peptide synthesis. The binding affinities of 
affibody molecules are considerably higher compared with the corresponding antibodies. The binding pocket of an affibody is composed of 13 amino acids, which can be randomized to bind a variety of targets. In contrast to monoclonal antibody, affibody has following advantages as a targeting ligand. First, the small size of affibody (MW: $6 \mathrm{kDa}$ ) guarantees its tissue/ cell penetration ability. Second, its functional end groups for chemical conjugation are distanced from its binding site. Moreover, affibody has a robust structure, and can be easily synthesized in a large-scale manner. All of these advantages make the affibody a valuable ligand for targeted drug delivery [Alexis et al., 2008; Manjappa et al., 2011]. Recently, antiHER2 affibody was also employed as a targeting ligand for nano-scaled drug delivery systems. Alexis et al. conjugated the anti-HER2 affibody to poly-(D, L-lactic acid)-poly (ethylene glycol)maleimide (PLA-PEG-Mal) copolymer for targeted delivery to cells that over-express the HER-2 antigen [Alexis et al., 2008].

\subsubsection{Aptamer targeting molecules}

A novel class of molecules, referred to as nucleic acid ligands (aptamers), has been developed to generate targeting agents. Aptamers are short single-stranded DNA or RNA oligonucleotides or modified DNA or RNA oligonucleotides that fold by intramolecular interaction into unique conformations with ligand-binding characteristics. Like antibodies, aptamers can be prepared to bind target antigens with high specificity and affinity. The use of aptamers as targeting molecules has several potential advantages over antibodies. Aptamers with high affinity for a target can be prepared through in vitro selection; a process called systemic evolution of ligands by exponential enrichment (SELEX) [Chai et al., 2011]. Conjugating aptamers to nanoparticles has shown to result in more efficient targeted therapeutics or selective diagnostics than non-targeted NPs. Farokhzad et al have developed $\mathrm{NP}$-aptamer (NP-Apt) conjugates that target the prostate specific membrane antigen (PSMA), a transmembrane protein that is up-regulated in a variety of cancers, using the A10 aptamer [Farokhzad et al., 2004]. This formulation has been further evaluated in vivo in a tumor model of LNCaP prostate cancer cells, which express PSMA antigens, and has been shown to regress tumor size effectively following a single intra-tumor injection over a 109-day study [Farokhzad et al., 2006].

\subsubsection{Oligopeptide-based targeting molecules}

Recently, a number of tumor homing peptides have been reported that specifically target cancer cells and show promising results for tumor targeted drug delivery. Peptides, being smaller than other targeting ligands, have excellent tissue penetration properties and can be easily conjugated to drugs and oligonucleotides by chemical synthesis. Peptides are nearly invisible to the immune system and are not taken up in the reticuloendothelial system like antibodies and so are expected to cause minimal or no side effects to bone marrow, liver, and spleen [Gu et al., 2007]. For example, Cilengitide ${ }^{\circledR}$ is a cyclic arginine-glycine-aspartic acid (RGD) peptide that binds to the cell adhesion integrin $\alpha_{\mathrm{v}} \beta_{3}$ on endothelial cells results in increased intracellular drug delivery in different murine tumor models and is currently in phase II clinical trials for the treatment of non-small cell lung cancer and pancreatic cancer. 
Despite the success mentioned, RGD-targeted therapy still encounters many challenges. First challenge is the limitation associated with the non-specific adhesive nature of the RGD-integrin targeting system. Integrins are extracellular receptors that are not only expressed on cancer cells but also on nearly all epithelial cells and are therefore not cancer specific. Recent development of phage display screening methods has successfully isolated peptide ligands with high specificity and affinity to cell-surface hormone receptors (LHRH receptors, somatostatin receptors) and tumor vasculature antigens [Gu et al., 2007]. One of those is a dodecapeptide identified through phage display by Zhang et al, referred to as peptide p160. Peptide p160 displays high affinity for the human breast cancer cell lines MDA-MB-435 and MCF-7 in vitro with very little affinity for primary endothelial HUVEC cells [Zhang et al., 2001]. Furthermore, in vivo bio-distribution experiments in tumor-bearing mice, p160 showed a higher uptake in tumors than in organs such as heart, liver, lung and kidney. Relative to the RGD-4C peptide, p160 showed high accumulation in tumor versus normal organs [Askoxylakis et al., 2005; Soudy et al., 2011; Zhang et al., 2001]. Despite the potential of peptide p160 as a potent tumor homing peptide, its applicability would be largely hindered by its instability toward proteases. To overcome this, peptides have to be chemically modified so that their blood clearance is minimized in comparison with their rate of uptake at the target sites. The most common strategies used to increase peptide proteolytic stability include introduction of D- or un-natural amino acids and peptide cyclization. In a recent study, Soudy et al have developed analogues of cancer targeting peptide p160 to improve proteolytic stability and maintain specific affinity for breast cancer cells. These analogues are potentially safe with minimal cellular toxicity and are efficient targeting moieties for specific drug delivery to breast cancer cells [Soudy et al., 2011].

\subsubsection{Growth factor or vitamin-based targeting molecules}

Growth factor or vitamin interactions with cancer cells represent a commonly used targeting strategy, as cancer cells over-express the receptors for nutrition to maintain their fast-growing metabolism. Epidermal growth factor (EGF) has been shown to block and reduce tumor expression of the EGF receptor, which is over-expressed in a variety of tumor cells such as breast and tongue cancer [Peer et al., 2007].

One of the most extensively studied small molecule targeting moieties for drug delivery is folic acid (folate). The high-affinity vitamin is a commonly used ligand for cancer targeting because folate receptors (FRs) are frequently over-expressed in a range of tumor cells. It has been used as a targeting moiety combined with a wide array of drug delivery vehicles including liposomes, protein toxins, polymeric NPs, linear polymers, and dendrimers to deliver drugs selectively into cancer cells using FR-mediated endocytosis [Gu et al., 2007]. The folic acidPEGylated PEI polyplex was evaluated as a gene carrier, and successfully delivered siRNA and pDNA to tumour cells [Kim et al., 2006].

Transferrin (Tf), an iron-binding glycoprotein interacts with Tf receptors (TfRs), which are overexpressed on a variety of tumor cells (including pancreatic, colon, lung, and bladder cancer) owing to increased metabolic rates. Direct coupling of these targeting agents to nanocarriers has improved intracellular delivery and therapeutic outcome in animal models. Tf- 
linked PEG-PEI was developed for tumor-selective gene delivery. The surface charge of the complexes was shielded by either PEG or a higher density of linked Tf to block undesired nonspecific interactions with blood components, followed by selective targeting to tumor cells. This approach resulted in a 100-fold higher gene expression in tumor cells compared with other tissues [Ogris et al., 2003].

One challenge with targeting receptors whose expression correlates with metabolic rate, such as folate and $\mathrm{Tf}$, is that these receptors are also expressed in fast growing healthy cells such as fibroblasts, epithelial and endothelial cells. Therefore, NP delivery system needs to be further refined and tuned to increase tumor selectivity [Gu et al., 2007; Peer et al., 2007].

\subsection{Intracellular delivery}

Non-viral vectors have to overcome multiple intracellular barriers subsequently after cellular uptake usually via endocytosis. After cellular internalization, a critical intracellular obstacle to non-viral gene delivery is degradation in the endosome/lysosome where nucleic acids are easily degraded by a mildly acidic $\mathrm{pH}$ and acid activated enzymes. The ability of non-viral vectors to release the nucleic acids in their intracellular targets, while surviving through low $\mathrm{pH}$ and digestive processes and avoiding unwanted premature decomplexation, is a key efficiency-determining requirement. The most widely believed theory in designing non-viral vectors for efficient endosomal escape is employing the "proton sponge effect" [Eliyahu et al., 2005]. Importantly, intracellular targets, where the nucleic acids are released from the vector, determine the overall gene delivery efficiency because the action sites are different for nucleic acid types. For example, plasmid DNA must be localized in the nucleus, where gene expression is initiated by transcription. However, nuclear entry is one of the major obstacles to non-viral gene delivery although nuclear translocation of plasmid DNA is crucial to achieve desired transfection efficiency. Moreover, the nuclear translocation mechanism of plasmid DNA in the cytoplasm has not yet been fully elucidated. Passive diffusion of macromolecules (e.g., plasmid DNA) in the cytoplasm is restricted by a complex network of microtubules, proteins, and various sub-cellular organelles. One explanation is the nuclear localization of plasmid DNA during cellular mitosis when the nuclear envelope disassembles. For non-dividing cells, the transport of polyplexes into the nucleus occurs only via an active transport mechanism through a nuclear pore complex that prevents molecules larger than $40 \mathrm{kDa}$ from passively diffusing into the nucleus. In contrast to plasmid DNA delivery, it is obvious that transnuclear localization of siRNA should be prevented to achieve efficient RNA interference (RNAi) since siRNA acts in the cytosol, where it targets mRNA with matching sequences [Shim and Kwon, 2012]. As nano-medicines often require delivery of their therapeutic payload to specific sub-cellular locations, knowledge about intracellular trafficking might prove useful for the control of the intracellular processing of nano-particles and lead to optimization of their design [Vercauteren et al., 2012].

\subsubsection{Escape from the endosomal compartment}

Following internalization of peptide-DNA condensates by endocytosis, the polyplex must be able to escape the endosome so that the DNA can be delivered to the nucleus for gene expression. After endocytosis, entrapment of the vector within the acidified vesicles of the endosomal/ 
lysosomal system is a critical barrier to non-viral gene delivery systems. Unfortunately, the environment of the lysosomal interior is harmful for nucleic acid (NA) integrity, unless the carrier offers sufficient protection against the degradation by the acid hydrolases [Vercauteren et al., 2012]. Viruses and some pathogenic bacteria have $\mathrm{pH}$-sensitive surface proteins that change conformation in mildly acidic environments such as in endosomes, and exhibit membrane-disruptive (fusogenic or endosomolytic) properties. Synthetic fusogenic peptides that mimic the sequences of these natural proteins have been confirmed to increase cytoplasmic gene delivery [Du et al., 2010]. Since escape from the endosomes is essential for efficient NA therapy, much attention has been paid to this issue, resulting in a variety of endosomolytic carriers. For example, cationic and $\mathrm{pH}$-responsive lipids can be added to phospholipid carriers to assist in releasing the NAs into the cytoplasm by destabilizing the endosomal lipid bilayer in the acidic environment of the endolysosomes. Another approach is based on the coupling of fusogenic peptides to the carriers, which undergo conformational changes after their exposure to the decreasing endosomal $\mathrm{pH}$ values, exposing hydrophobic faces of the fusion peptide which destabilizes the endosomal membrane. Examples of these endosome-disruptive peptides are the influenza HA2 peptide, melittin, the T-domain of the diphtheria toxin or the GALA peptide [Vercauteren et al., 2012]. In an effort to translate the proton sponge activity to gene delivery peptides, histidine has been added to peptide sequences. The imidazole group of histidine has a pKa of $\sim 6.0$, therefore allowing it to become protonated in the acidic environment of the endosome. At physiological $\mathrm{pH}$ the histidines will remain neutrally charged, thereby imparting selective membrane disruption in the acidic endosome. In the past two decades, synthetic $\mathrm{pH}$-sensitive polymers as endosomolytic agents have attracted great interest due to their low or non immunogenicity, which is a concern of using fusogenic proteins or peptides. Polymers such as PEI contain several secondary amines that are easily protonated in the acidic environment of the endosome. As protons are pumped in, PEI absorbs the protons leading to endosomal swelling and membrane disruption. In general, these smart polymers have both hydrophobic parts and weakly acidic groups, which afford them $\mathrm{pH}$-dependent endosomolytic properties. At physiological $\mathrm{pH}$, the polymers have little endosomolytic activity but undergo a conformational change at endosomal $\mathrm{pH}$ and show membrane-disruptive properties. This provides the polymers with reduced toxicity to the other biomembranes at neutral $\mathrm{pH}$, but with the ability to facilitate endosomal escape [Fig. 1A; Du et al., 2010].

\subsubsection{Cytosolic un-packaging}

The final step of the gene delivery process, un-packaging of the polyplex, can limit the efficiency of gene delivery and expression. For in vivo polyplex gene delivery, the polycation condenses the DNA to protect it and facilitate its entry and passage through target cells. However, once inside the nucleus, in order to be processed by RNA transcription complexes, the DNA may first need to dissociate from the polycation. Strong binding of the polycations to the nucleic acids may limit the intracellular un-packing of the polyplexes which is necessary for an efficient transfection. Although some viruses have evolved highly specific and intensive mechanisms for uncoating within the cell, a synthetic polycation may not release DNA with similar high efficiency [Schaffer et al., 2000]. One of the solutions to these problems is to use degradable polycations. Although the degradable polycations whose degradation is based on 
the hydrolysis of an ester or amide bond have been widely used as gene carriers with decreased cytotoxicities, it is difficult to control the degradation occurring in the cytoplasm where free siRNA should be released to take action. Since the reduction potential in the cytoplasm is much higher (100 fold) than in the extracellular environments, a promising strategy to create an interactive delivery system is to exploit the redox gradient between the extra- and intra-cellular compartments, reduction-sensitive polyplexes are considered to be superior degradable candidates, especially for siRNA delivery [Bauhuber et al., 2009; Du et al., 2010].

\subsubsection{Nuclear import}

The nuclear envelope that separates the cell's genetic material from the surrounding cytoplasm represents a physical barrier for nuclear import of macromolecules such as pDNA. The nuclear envelope contains openings in the form of nuclear pore complexes, which allow free diffusion of molecules up to $50 \mathrm{kDa}$, corresponding to a hydrodynamic diameter of approximately 10 $\mathrm{nm}$. There are several lines of evidence showing that nuclear import is a rate-limiting step for transfection of pDNA. Nuclear import of pDNA may be more challenging for transfection of non-dividing cells. Indeed, non-dividing cells showed a $90 \%$ lower expression level as compared to actively dividing cells. Strategies for nuclear import of genes have been developed following further elucidation of the endogenous nuclear import machinery [Wang et al., 2011]. In nature, large molecules with sizes up to $30 \mathrm{~nm}$ in diameter that require trans-nuclear transport contain nuclear localization signals (NLS) that are recognized by nuclear transport receptors like importins or transportins, and the whole complex is thereafter actively transported through NPCs [Vercauteren et al., 2012]. The nuclear localization sequence is a major player that shuttles protein-plasmid complexes through the nuclear pore. NLS-mediated active nuclear translocation involves a process starting from its interaction with cytoplasmic importins to binding of the NLS to the nuclear pore complex and the passage through the pore. Identification of the NLSs, such as SV40 from the larger tumor antigen Simian virus 40 and M9 from nuclear ribonucleoprotein, enabled design of non-viral gene vectors with nuclear targeting properties [Wang et al., 2011].

\section{Designing switchable nanosystems for medical application}

To enhance therapeutic efficacy while minimizing side effects, a large number of nanomaterial based platforms have been developed that allow simple delivery of genes. Based on important earlier work in the field of liposomal gene delivery and inorganic nanomaterials, the last decade has brought a broad array of new and improved nanoscale carrier platforms such as biodegradable and non-degradable polymers, dendrimers, carbon nanotubes, metallic and organic nanoparticles, quantum dots, nanogels or peptidic nanoparticles. Ideally, gene vectors should be capable of self-assembly with nucleic acids and accommodate with any type of nucleic acid or their combination. They should also target cells of interest, escape from endosomes and/or transport into nuclei. A viable gene vector for systemic delivery needs to minimize toxicity and phagocytosis and avoid nonspecific interactions and self-aggregation. For potential gene delivery applications, ideal 
gene carriers need to combine both the targeting property and the stimulus responsiveness to enhance the bioavailability of the gene as well as to reduce the side effects. Therefore, designing stimulus-responsive nanoparticles for programmed gene delivery, which release the gene on arrival at the targeted site, is highly desired. Stimulus-responsive nanoparticles produce physical or chemical changes when subjected to external signals, including variations of macromolecular structures, solubility, surface properties, swelling and dissociation [Lehner et al., 2012]. Stimulus-responsive nanoparticles can be classified based on the type of stimulus as internally and externally controllable materials. Internal stimuli (e.g. activation by $\mathrm{pH}$, redox potential, enzymes) might be controlled by a molecular mechanism highly specific for a disease and therefore improve on targeting properties. However, absolutely disease-specific internal molecular triggers are difficult to find for certain diseases. External stimuli like light, ultrasound, electromagnetic fields or ionizing radiation have the advantage of being focusable on certain body areas. This may be a significant advantage where a target cell is strongly involved in pathogenesis at one location (e.g., cancer stem cells in a cancer tissue), but of vital importance in other locations (e.g., stem cells in the bone marrow). A key challenge in externally controlled nanomaterials is tissue penetration and avoidance of undesired tissue damage in the radiation path from radiation source to target tissue. The ease of temporal control in external stimuli may represent a particular advantage for certain applications [Lehner et al., 2012]. Herein, we focus on the use of internal and biological stimuli that can be used to incorporate switch functionality into such nanocarriers and describe the clinical experience with various nanosize carrier systems as a basis for the design of new, improved, functional and "intelligent" nanosystems for gene delivery.

\section{1. $\mathrm{pH}$ differences for stimuli-responsive delivery}

The main property that is the basis of utilization of $\mathrm{pH}$ responsive polymers in gene delivery is the significant change in $\mathrm{pH}$ value within the cellular compartments. There are numerous $\mathrm{pH}$ gradients in physiological and pathological processes [Du et al., 2010]. The $\mathrm{pH}$ profile of pathological tissues, such as inflammation, infection and cancer, is significantly different from that of the normal tissue. The $\mathrm{pH}$ at systemic sites of infections, primary tumors, and metastasized tumors is lower than the $\mathrm{pH}$ of normal tissue. The $\mathrm{pH}$, surface charge and density of low density lipoprotein receptors are the factors that show notable differences among the normal and tumor tissues. All these properties are known to influence the drugs' physicochemical properties and are exploited for enhanced delivery to the target site. The extracellular $\mathrm{pH}$ values of solid tumor are in general slightly lower than in blood or other normal tissues. Since tumors proliferate very rapidly, the vasculature of tumor is often insufficient to supply enough nutritional and oxygen needs for the expanding population of tumor cells. This results in difference in metabolic environment between the various solid tumors and the surrounding normal tissue. The insufficient oxygen in tumor leads to hypoxia and causes production of lactic acid and hydrolysis of ATP in an energy-deficient environment contributes to an acidic microenvironment, which has been found in many tumors. Most of the solid tumors have lower extracellular $\mathrm{pH}(6.5)$ than the surrounding tissues $(\mathrm{pH}=7.5)$. The $\mathrm{pH}$ is compartmentalized in tumor tissue into an intracellular component (pHi), which is similar in tumor and normal tissues and an extracellular component $(\mathrm{pHe})$, which is relatively acidic in tumors 
[Ganta et al., 2008]. This behavior can be utilized for the preparation of stimuli responsive drug or gene-delivery systems, which can exploit the biochemical properties at the contaminated site for targeted delivery. Cellular components such as the cytoplasm, endosomes, lysosomes, endoplasmic reticulum, golgi bodies, mitochondria and nuclei are known to maintain their own characteristic $\mathrm{pH}$ values. It is well known that the lower $\mathrm{pH}$ values are found in endosomes (5.5-6.0) and lysosomes (4.5-5.5). On the basis of these discoveries, various $\mathrm{pH} / \mathrm{acid}-$ sensitive polymers have been developed as carriers for pDNA, ODN or siRNA delivery [Du et al., 2010; Ganta et al., 2008].

\subsubsection{Low $\mathrm{pH}$-sensitive reversible shielding/masking}

This part focuses on the design and synthesis of polymeric carriers that can condense a large dose of therapeutic nucleic acids into particles that can sense the differences in environmental $\mathrm{pH}$. The $\mathrm{pH}$-modulated or self-regulated polymers are designed to use these $\mathrm{pH}$ differences by incorporating appropriate structural or functional features into the basic scaffold of the polymers to improve the efficacy of gene delivery. In general, polycations (PCs), such as PEI or dendrimers (polyplexes) or with cationic lipids (lipoplexes) with positively charged surfaces are preferred for gene delivery in cell culture [Du et al., 2010]. Although, these positively charged particles are favorable for gene transfer efficiency in vitro, they are problematic for systemic gene targeting. Upon systemic application of positively charged cationic lipid based formulations (CL), polycations and lipopolyplexes may result in significant toxicity and/or poor efficiency due to plasma protein binding, interaction with blood cells or activation of the complement system and therefore has limited their application for in vivo uses via systemic administration [Du et al., 2010; Walker et al., 2005]. In general, the toxicity of cationic polymers increases with molecular weight, branched polymer morphology, and cationic charge density. A common approach for masking the surface charge of polyplexes is to coat particles with a hydrophilic polymer such as polyethylene glycol (PEG). Shielding the cationic surface by PEGylation and tailoring cationic density and polymer morphology have been popularly exploited to reduce the cytotoxicity [Shim and Kwon, 2012]. PEGylation of polyplexes prevents their aggregation, lowers toxicity, increases circulation time and improves systemic targeted gene transfer. Unfortunately, at the same time as shielding improves the properties of polyplexes for systemic application it appears to reduce its cell transfection activity due to two important barriers such as reduced cellular uptake and inadequate release of the transported gene at the target cells. The cellular uptake has been enhanced by attaching targeting ligands to the polyplexes, however, the transfection efficiency of the targeted PEG vectors often still does not reach the level of the uncoated gene vectors. This suggests that the stable shield may also hinder intracellular gene transfer steps following endosomal uptake. Therefore, it is clear that to develop an optimal nonviral system for systemic application it must have a more dynamic character [Du et al., 2010] Its surface charge must be neutralized during circulation but after reaching its target cell, the cationic surface charge should be re-exposed for efficient gene transfer. In order to solve these conflicting issues, $\mathrm{pH}$-sensitive reversible shielding or masking strategies have been developed through $\mathrm{pH}$-sensitive PEGylation of lipid- or polymer-based carriers as the PEG shield intended to be removable in intracellular 
endosomes or in the slightly acidic extracellular microenvironment of tumors. Lipoplexes and polyplexes containing a $\mathrm{pH}$ cleavable PEG shield were found to be less effective in gene transfer than the corresponding stably shielded particles. There is a strategy to overcome this challenge. The neutralizing shield is attached to the DNA polyplex core via an acid-labile linkage forming a shielded particle for systemic circulation. Chemical linkages that may display $\mathrm{pH}$-dependent hydrolytic degradation, once internalized into endosomal and lysosomal compartments include acetal-ketal linkage, vinyl ether, orthoesters, and hydrazones. Under the acidic environment of the endosomal compartment, these linkages undergo acid-induced hydrolysis and thereby trigger deshielding of the polyplex core. Therefore, the acid-labile bioreversible shielding polymer exerts a significant impact on the outcome of transfection efficiency [Du et al., 2010].

Walker and colleagues reported reversible shielding of polyplexes with pH-triggered deshielding properties, enabled by conjugating PEG to the polycations poly-L-lysine (PLL) and PEI via a pH-sensitive hydrazone bond (PC-HZN-PEG) of acyl hydrazides or 2-pyridyl hydrazines. The reversibility of the hydrazone bond within conjugates was determined at physiological and endosomal acidic $\mathrm{pH}$, identifying suitable linker systems for endosomal deshielding. The polyplexes with the acid-sensitive linkages showed much higher plasmid gene delivery efficiencies (1-2 orders of magnitude) than those with stable linkages, both in vitro and in vivo [Walker et al., 2005].

Sawant et al. demonstrated that the use of a lowered pH-degradable PEG-Hz-PE produced particles (polyethylene glycol-phosphatidylethanolamine conjugates) with transfection activity sensitive to changes in $\mathrm{pH}$, which has a promise for site-specific transfection of tumor cells in vivo. In this study, the encapsulation of PEI-PE/DNA complexes into $\mathrm{pH}$-sensitive micelle-like PEG-Hz-PE coat increased the stability of DNA in complete medium and increased transfection efficiency by being responsive to changes in $\mathrm{pH}$. In vivo, the PEG2000-Hz-PE is expected to shield the PEI-PE/DNA complex in the systemic circulation and expose the complex only at the tumor sites where the $\mathrm{pH}$ is slightly acidic and can facilitate the removal of the PEG coat [Sawant et al., 2012].

Murthy et al. synthesized 'encrypted' polymeric carrier that consisted of hydrophobic, membrane disruptive methacrylate polymers onto which hydrophilic PEG chains have been grafted through acid-degradable acetal linkages; a $p$-aminobenzaldehyde acetal linkage demonstrated a suitable hydrolysis profile at endosomal pH [Murthy et al., 2003].

In 2007, Knorr et al. synthesized a new PEGylation reagent containing p-piperazinobenzaldehyde acetal linkage and a maleimide moiety which can be coupled to thiol-functionalized compounds. For reversible shielding of polyplexes, PEG-acetal-maleimide (MAL) was conjugated to PEI. At $37^{\circ} \mathrm{C}$, the PEG-acetal-PEI conjugate were found to be shielded and stable. In contrast, at endosomal $\mathrm{pH}$, the particles were deshielded and aggregated within $0.5 \mathrm{~h}$. The reversibly shielded (PEG-acetal-PEI) polyplexes were found to have approximately 10-fold enhanced gene transfer efficiency than stable shielded polyplexes when tested on the two different cell lines, Renca-EGFR cells and K562 cells [Knorr et al., 2007]. 
Recently, Sethuraman et al. have developed pH-Responsive Sulfonamide/PEI nanoparticles that effectively target the acidic extracellular matrix of tumors, which shows a sharp $\mathrm{pH}$ profile, was able to shield positively charged complexes at physiological $\mathrm{pH}$ of 7.4. The $\mathrm{pH}$ sensitive polymer was able to detach from the complex when the $\mathrm{pH}$ environment decreased to $\mathrm{pH}=$ 6.6. The polymeric nanoparticle formed through electrostatic attraction is designed in such a way that the final particle is neutral. The polyplexes formed by PEI and pDNA were coated electrostatically with an ultra $\mathrm{pH}$-sensitive diblock copolymer, poly (methacryloyl sulfadimethoxine)-b-PEG. The central idea of this design is that when the particles experience a decrease in $\mathrm{pH}$ as they extravasate into tumor tissue due to enhanced permeability and retention effect, the sulfonamide groups would lose their charge and get detached from the carrier complex. Most of the carriers developed so far do not have the high sensitivity that is required to respond to such small differences in $\mathrm{pH}$ between tumors and normal tissues. This is because the carboxylic acid based polymers show transitions in about one $\mathrm{pH}$ unit which is very broad, and that transition is much below the physiological and tumor $\mathrm{pH}$ range, whereas the poly(methacryloyl sulfadimethoxine) (PSD)-block-PEG PSDb-PEG polymer shows transition within $0.2 \mathrm{pH}$ units between the physiological and tumor $\mathrm{pH}$. These sulfonamide polymers are able to distinguish the small difference in $\mathrm{pH}$ between normal and tumor tissues and hence has remarkable potential in drug targeting to tumor areas [Sethuraman et al., 2008].

\subsection{2. pH-dependent endosomolytic polymers}

Following internalization of lipoplexes or polyplexes via the endocytic pathway, endosomal entrapment and subsequent lysosomal degradation are a major blockage that limits the efficiency of gene delivery. After cellular uptake of the gene carriers, the carrier should escape from the endosomal compartment in order to reach the cytosol and then, the nucleus. This requires dissociation of the internalized carrier from the receptors that triggered the internalization process. In addition, the membranes of the endosomes should be destabilized to allow translocation of the carriers into the cytosol [Mastrobattista et al., 2006]. Identification of endosomolytic or fusogenic components and their integration into non-viral gene delivery systems are major strategies being exploited to facilitate endosomal escape.

In the case of polyplexes, PEI and polyamidoamine (PAMAM) are two representative cationic polymers with a high efficiency of gene transfer due in part to their capability to facilitate endosomal escape. A "proton sponge effect" provides a sound explanation for the intrinsic endosomolytic activity. Upon PEI-based or PAMAM-based polyplex entry into acidic endosomes, the polymer behaves as a sponge that absorbs protons as a result of protonation of the polymer-containing amine groups (primary, secondary and tertiary). Accumulation of protons subsequently drives an influx of counter chloride ion into endosomes, leading to increased osmotic pressure and subsequent flow of water into the endosomal interior and eventually swells and ruptures endosomal membrane [Eliyahu et al., 2005].

PEIs are available in a wide range of molecular weights (MW) from $423 \mathrm{Da}$ to $800 \mathrm{kDa}$ and with different branching degree (from linear to branched). Generally, high MW, branched PEIs have high transfection efficiency but also high toxicity due to high cationic charge. By contrast, low MW PEIs are less toxic but less efficient as gene delivery agents. Many efforts have been 
directed towards creating PEI derivatives combining higher transfection efficacy and good biocompatibility. One approach to reduce the cytotoxicity, biodegradable polyethylenimine with imine linkages as acid-labile moieties were synthesized and investigated for pDNA delivery. The half-life of the acid-labile PEI was $1.1 \mathrm{~h}$ at $\mathrm{pH}=4.5$ and $118 \mathrm{~h}$ at $\mathrm{pH}=7.4$, suggesting that the acid-labile PEI may be rapidly degraded into non-toxic low molecular weight PEI in acidic endosome. Acid-labile PEIs showed close transfection efficiency to PEI 25KDa, but much less toxicity due to the degradation of acid-labile linkage. Therefore, the acid-labile PEIs may be useful for the development of a non-toxic polymeric gene carrier [Kim et al., 2005].

The cationic lipids have been shown to destabilize the endosomal membrane. Addition of dioleoyl phosphatidylethanolamine (DOPE) or cholesteryl hemisuccinate (CHEMS) to nonviral vectors affected the $\mathrm{pH}$-sensitivity of the formulations. The mechanism behind the phenomenon, is that electrical interaction between cationic lipids and anion endosomal membranes results in the formation of ion-pairs that promote the formation of the inverted hexagonal (HII) phase and disrupt endosomal membrane. Many preparations of lipoplexes contain DOPE as a helper lipid for fusogenic functionality. The ability of DOPE to destabilize endosomal membranes is based on its propensity to acquire an inverted hexagonal phase (HII). DOPE has a small cross-section head group and a large hydrocarbon area that favors a nonbilayer structure with a cone shape that facilitates the destabilization of endosomal membranes and gene transfection. The $\mathrm{pH}$-responsive component, CHEMS, being negatively charged at neutral $\mathrm{pH}$ stabilizes the bilayer structure, however, at acidic $\mathrm{pH}$ it becomes protonated and loses its stabilization property [Ma et al., 2007; Wu and Zhao, 2007].

Another approach to reduce the cytotoxicity of PEI is modification with hydrophobic moieties, such as lipids. Since lipids are the main component of cell membrane, modification with hydrophobic moieties may result in additional hydrophobic interaction between polyplexes and cell membranes, which in turn would facilitate the delivery of a payload into cells. Various hydrophobic modifications have been tried, including modification with cholesterol, myristate, dodecyl iodide, hexadecyl iodide, palmitic acid, oleic acid, stearic acid, and phosphatidylcholine. In a recent study, Sawant et al. developed the synthesis and characterization of a PEI-DOPE conjugate, which was explored for gene delivery in vitro. The modification with DOPE strongly increased the transfection efficiency of low molecular weight PEI-1.8 kDa without any negative effects on its low cytotoxicity. The PEI-PE conjugate was synthesized by reacting a phospholipid with low molecular weight PEI (PEI-1.8). It was assumed that a PEI-PE conjugate would condense DNA due to the electrostatic interaction between polycationic PEI moieties, while the lipid moieties would help to increase cell interaction of the complexes and facilitate their incorporation into lipid-based micellar systems via hydrophobic interactions [Sawant et al., 2012].

In order to take the advantages of both polycationic polymers and liposomes, Nie et al. have constructed programmed lipopolyplexes, featuring well compacted DNA by PEI and liposome complexing. Liposomes contain the helper lipid DOPE and a pH-cleavable PEG-hydrazonecholesterol conjugate for shielding. Lipopolyplexes composed of DNA condensed with PEI, phospholipids including dioleoyl phosphatidyl ethanolamine (DOPE) and pH-labile $\omega$-2pyridyldithio polyethylene glycol $\alpha$-succinimidylester (OPSS)-PEG-HZN-Chol and yielded 
particles of $160 \mathrm{~nm}$ size and a zeta potential of $+7 \mathrm{mV}$. Pyridylhydrazone-based Chol-PEG was included in the liposomes for shielding in extracellular compartments and dynamic deshielding in acidic conditions such as in endosomes. In addition, this cholesterol-PEG derivative contained also a pyridyldithio moiety to provide the possibility of coupling thiol-functionalized compounds, such as receptor ligands [Fig. 1B.; Nie et al., 2011].

The endosome-escape potential of poly-histidine increases their use for delivery of nucleic acids. The imidazole ring within histidine is a major component. Under the action of an acidic endosomal interior, the weak basic nature of the imidazole ring with pKa around 6 allows its protonation and acquires cationic charges which trigger the destabilization of endosomal membranes. Accumulation of histidine residues within endosomes could elicit a proton sponge effect and destroy endosomes as a result of their increased osmolarity. Both chemistry conjugation and genetic engineering have produced a series of histidine-rich polymers and peptides as well as lipids with imidazole, imidazolinium or imidazolium polar heads. These histidylated carriers have been used to deliver nucleic acids including pDNA, mRNA or siRNA duplex in vitro and in vivo with increased transfection efficiency [Midoux et al., 2007; Martin and Rice, 2007].

The histidine-rich peptide H5WYG is a derivative of the N-terminal sequence of the HA-2 subunit of the influenza virus hemagglutinin in which five of the amino acids have been replaced with histidine residues. H5WYG is able to selectively destabilize membranes at a slightly acidic $\mathrm{pH}$ as the histidine residues are protonated. An anionic derivative of this peptide, E5WYG, in which the histidines are replaced by glutamic acid residues, is completely ineffective at membrane permeabilization at a $\mathrm{pH}=6.8$, while H5WYG can disrupt $50 \%$ of cells at $\mathrm{pH}=6.8$ and $97 \%$ of cells at $\mathrm{pH}=6.2$ within 15 minutes. H5WYG was also able to retain its activity in the presence of serum, making it possible for use in vivo. The most well-known non-viral DNA condensing agent is poly-L-lysine. However, PLL only exhibits modest transfection when used alone and requires the addition of an endosomolytic agent such as chloroquine or a fusogenic peptide to allow for release into the cytoplasm. To increase the transfection efficiency of polylysine without the addition of membrane-disrupting agents, a histidine-substituted polylysine can be constructed that is able to become cationic at endosomal pH [Martin and Rice, 2007].

In an attempt to facilitate endosome escape, many strategies have been developed to mimic the viral mechanism for endosome destabilization. Viruses have acquired efficient solutions for escaping from the maturating acidifying endosomes. For example, glycoproteins of enveloped viruses contain hidden fusion peptides which are exposed after endocytosis, to trigger fusion of the viral with the endosomal membrane [Wagner, 2011]. It is well-established that the influenza virus utilizes the $\mathrm{pH}$-sensitive membrane-destabilizing Hemagglutinin protein (HA2) displayed on the viral coat to disrupt the endosomal membrane and enter the cytoplasm. Hemagglutinin and other fusion proteins are characterized by their unique ability to switch from an ionized and hydrophilic conformation at physiologic $\mathrm{pH}$ to a hydrophobic and membrane-active one in response to acidic endosomal $\mathrm{pH}$ gradients, which destabilizes the endosomal membrane leading to leakage of endosomal contents into the cytoplasm [Lin et al., 2010]. At neutral pH, the HA2 subunit adapts a non-helical conformation due to charge 
repulsion arising from ionization of glutamic and aspartic acid residues. Within the interior of the acidic endosomal compartment, however, the HA2 subunit transitions into a stable helical secondary structure due to the protonation of glutamic and aspartic acids. The hydrophobic and hydrophilic faces of the helical conformation favor endosomal membrane destabilization.

Endocytosed non-enveloped viruses such as rhinovirus or adenovirus expose lytic domains which directly disrupt the endosomal membrane, either (in case of rhinovirus) generating a pore large enough for crossing of the viral RNA strand into the cytoplasm or (in case of adenovirus) disrupting the whole endosome. Such lytic domains have been utilized in artificial settings as synthetic peptides for endosomal escape of polyplexes [Wagner, 2011].

Synthetic peptides mimicking a virus's fusogenic peptides have also been designed for delivery of nucleic acids. The amphipathic peptide, GALA, was synthesized with 30 amino acid residues with a repeated amino acid sequence (e.g., glutamic acid-alanine-leucinealanine) that demonstrated $\mathrm{pH}$ sensitive fusogenic properties. A GALA peptide with a formulation based on DNA/cationic liposome/ Transferrin (Tf) complexes induced enhanced gene transfection. It is assumed that Tf-triggered internalization of the complexes by receptormediated endocytosis and the GALA peptide promoted endosomal destabilization and release of the genetic material into the cytoplasm [Park et al., 2010].

Modification of multifunctional envelope-type nano-devices (MENDs) with GALA peptide facilitating endosomal escape, leads to the enhanced transfection efficiency of pDNA and siRNA duplex in vitro and in vivo. To mimic envelope-type virus-like delivery systems, Sasaki et al. developed an artificial nanocarrier system termed as a MEND, which consists of a condensed DNA nanoparticle and lipid envelope which is further equipped with Tf, Cholesterol-GALA (Chol-GALA), or PEG-GALA to achieve target specificity and controlled intracellular trafficking, especially endosomal escape. As GALA can show fusogenic activity only at acidic $\mathrm{pH}$, the direct fusion of MEND with the plasma membrane is feasible only after internalization. The Tf-MEND introduced with Chol-GALA or PEG-GALA showed a ten-fold higher transfection than that displayed by Tf-MEND. However, the simultaneous introduction of Chol-GALA and PEG-GALA enhanced the transfection efficiency more than 100-fold as compared to Tf-MEND mediated transfection. Chol-GALA and PEG-GALA operated synergistically to destabilize the envelope and endosomal membranes, respectively. Chol-GALA interacted with the envelope membrane whereas PEG-GALA penetrated into the endosomal membrane, which could destabilize the membranes and induce fusion [Sasaki et al., 2008].

A cationic counterpart of GALA is the peptide KALA which is formed by substitution of the alanine of GALA with lysine and a decrease in content of glutamic acid. KALA was the first designed peptide that could bind DNA, destabilize membranes, and mediate significant gene delivery. KALA undergoes a $\mathrm{pH}$-dependent amphipathic $\alpha$-helix to random coil conformational change, when the environmental $\mathrm{pH}$ decreased from 7.5 to 5.0. KALA can deliver both ODN and pDNA into cells [Park et al., 2010].

Several groups have focused their efforts on the development of synthetic, polymeric carriers that mimic the endosomolytic properties of fusogenic proteins and enhance the cytoplasmic delivery of therapeutic macromolecules. Recently, the ability of natural phospholipids to self- 
assemble into organized membrane-enclosed structures has been mimicked by amphiphilic co-polymers. The combination of a hydrophobic monomer and an ionizable co-monomer that has a more hydrophilic nature is one of the interesting strategies that have been adopted frequently for $\mathrm{pH}$-responsive gene delivery. A change in $\mathrm{pH}$ and subsequent adjustment in the net charge causes the phase transformation depending on the hydrophobic and hydrophilic balance of the copolymer. These synthetic amphiphiles form assemblies that are remarkably similar to biological analogues, such as vesicles. Polymeric amphiphiles have much higher molecular weights than phospholipids and can self-assemble into more entangled membranes, imparting improved mechanical properties to the final structure [Park et al., 2010]. Polymer vesicles or polymersomes can combine several different polymeric compositions provided that they have the correct hydrophile/hydrophobe ratio. Particularly interesting for biomedical applications, are those copolymers that combine hydrophobic blocks with the non-antigenic properties of either PEG or biomimetic poly (2-(methacryloyloxy) ethyl phosphorylcholine) (PMPC). Indeed, the macromolecular nature of such polymersomes offers several advantages as compared to low-molecular-mass amphiphilic systems such as liposomes. PEG or PMPC polymersomes can be decorated by denser and higher molecular mass hydrophilic polymeric corona, with consequent longer circulation times than more traditional delivery systems such as stealth liposomes and other nanoparticles. Typical examples are the copolymers of methyl methacrylate (MMA) with methacrylic acid (MAc) or dimethylaminoethyl methacrylate (DMAEMA). The MMA is the hydrophobic section while MAc is the hydrophilic part of the chains. MAc is more hydrophilic at high $\mathrm{pH}$ when the carboxylic groups $(\mathrm{COOH})$ are deprotonated, but becomes more hydrophobic when the carboxylic groups are protonated. The phase change occurs around the pKa value of the carboxylic groups, which is around 4.5-5.5. The copolymers of MMA with DMAEMA, which are hydrophilic at low $\mathrm{pH}$, when the amino groups are protonated, but more hydrophobic when the amino groups are deprotonated. The key feature of these polymers is their ability to directly enhance the intracellular delivery of DNA, by destabilizing biological membranes in response to $\mathrm{pH}$ changes within the vesicular compartment [Park et al., 2010].

These copolymers are characterized by their unique ability to "sense" the changes in environment $\mathrm{pH}$ where they undergo a change from a hydrophilic, stealth-like conformation at physiologic $\mathrm{pH}$ to a hydrophobic and membrane-destabilizing one in response to acidic endosomal pH gradients [Lin et al., 2010]. Poly(ethylacrylic acid) (PEAA) is the first reported polymer to display a $\mathrm{pH}$-dependent disruption of synthetic lipid vesicles at acidic $\mathrm{pH}=6.3$ or lower. The family of poly (alkyl acrylic acid) like poly (methyl acrylic acid) (PMAA), poly (ethyl acrylic acid) (PEAA), poly (propyl acrylic acid) (PPAA), and poly (butyl acrylic acid) (PBAA) were provided with $\mathrm{pH}$-dependent, membrane destabilizing activities. These polymers are hydrophilic and stealth-like at physiological $\mathrm{pH}$, but become membrane-destabilizing after uptake into the endosomal compartment where they enhance the release of therapeutic cargo into the cytoplasm [Lin et al., 2010].

Diblock copolymers PMPC-PDPA as biomimetic polymersomes were used for gene delivery made of $\mathrm{pH}$-sensitive poly (2-(methacryloyloxy) ethyl-phosphorylcholine)-co-poly (2(diisopropylamino) ethyl-methacrylate) (PMPC-PDPA) diblock copolymers. The high bio- 
compatibility features are ascribed to PMPC residue whereas the PDPA block imparts $\mathrm{pH}$ responsiveness. These diblock copolymers exist as stable vesicles at physiological $\mathrm{pH}$ but these vesicles rapidly dissociate at around $\mathrm{pH}=5-6$ to form unimers. The phase transition of vesicles to unimers takes place because of protonation of the tertiary amine groups of PDPA chains, which transforms hydrophobic PDPA chains into a hydrophilic entity from physiological $\mathrm{pH}$ to mild acidic conditions. pH-responsive PMPC-PDPA vesicles release their contents upon exposure to the low $\mathrm{pH}$ (5.5) media in endosomes or lysosomes [Lomas et al., 2007].

Comb-like diblock copolymers with a robust membrane-destabilizing activity in response to a mildly acidic $\mathrm{pH}$ in the endosome were also developed. Acid-cleavable hydrazone linker has also been frequently utilized to achieve the facilitated release of nucleic acids from polyplexes. The first $\mathrm{pH}$-sensitive block was the copolymer of ethyl acrylic acid (EAA) and hydrophobic methacrylate, and the second block was the copolymer of hexyl methacrylate (HMA) and trimethyl aminoethyl methacrylate (TMAEMA) grafted with Nacryloxy succinimide or $\beta$-benzyl L-aspartate $\mathrm{N}$-carboxy-anhydride via acid-cleavable hydrazone linkages. These comb-like polymers exhibited a high concentration-dependent hemolytic activity in acidic solutions and degraded into smaller fragments via acid-hydrolysis of hydrazone linkages, resulting in minimized toxicity and facilitated elimination by renal excretion in vivo. These polymers formed siRNA complexing polyplexes that were stable even in the presence of serum and nucleases, and efficiently silenced GAPDH expression in MCF-7 breast cancer cells in vitro [Lin et al., 2010].

\subsubsection{Low pH-sensitive siRNA/ODN-polymer conjugates}

Small interfering RNA (siRNA)-based therapies have great potential for the treatment of diseases such as cancer, but an effective delivery strategy for siRNA is unclear. Benoit et al. developed a ternary $\mathrm{pH}$ responsive endosomolythic complex for the delivery of siRNA in order to sensitize drug-resistant ovarian cancer cells to doxorubicin. The electrostatic complexes were self-assembled by cationic micelles used as a nucleating core, siRNA and a $\mathrm{pH}$-responsive endosomolytic polymer. Cationic micelles were formed from diblock copolymers of dimethylaminoethyl methacrylate (pDMAEMA) and butyl methacrylate $(\mathrm{pDbB})$. The hydrophobic butyl core mediated micelle formation while the positively charged pDMAEMA corona enabled siRNA condensation. To enhance cytosolic delivery through endosomal release, a $\mathrm{pH}$-responsive copolymer of poly (styrene-alt-maleic anhydride) (pSMA) was electrostatically complexed with the positively charged siRNA/micelle to form a ternary complex. Complexes exhibited size (30-105 nm) and charge (slightly positive) properties and mediated uptake in $>70 \%$ of ovarian cancer cells after $1 \mathrm{~h}$ of incubation [Benoit et al., 2010]. The optimized formulation of the resulting ternary nano-vector were used to deliver siRNA against polo-like kinase 1 ( $p l k 1)$, a gene up-regulated in many cancers and increased doxorubicin sensitivity in the drug-resistant ovarian cancer cells. Sensitization occurred through a p53 signaling pathway, as indicated by caspase 3/7 upregulation following plk1 knockdown and doxorubicin treatment, and this effect could be abrogated using a p53 inhibitor. To demonstrate the potential for dual delivery from this polymer system, micelle cores were subsequently loaded with doxorubicin and utilized in 
ternary complexes to achieve cell sensitization through simultaneous siRNA and drug delivery from a single carrier. These results show that knockdown of $p l k 1$ results in sensitization of multi-drug resistant cells to doxorubicin, and this combination of gene silencing and small molecule drug delivery may prove useful to achieve potent therapeutic effects [Benoit et al., 2010].

Acid-degradable and targetable polyion complex (PIC) micelles increased the gene silencing in hepatoma cells. This multi-functional carrier was synthesized by assembling lactosylatedPEG-siRNA conjugates via acid-labile $\beta$-thiopropionate linkages into PIC micelles through the mixing with poly (L-lysine). The lactosylated-PEG-siRNA/PLL polyplexes were successfully transported into hepatoma cells in a receptor-mediated manner, releasing hundreds of active siRNA molecules into the cellular interior responding to the $\mathrm{pH}$ decrease in the endosomal compartment. This carrier exhibited almost 100 times enhancement in gene silencing activity and facilitating the practical utility of siRNA therapeutics [Oishi et al., 2005].

\subsection{Redox-responsive nanocarriers}

One of the several micro-environmental features, which have been widely exploited for improving the efficiency of nucleic acid delivery, is the redox potential gradient existing between extracellular environment and various subcellular organelles in normal as well as pathological states. The existence of a high redox potential gradient between oxidizing extracellular space and the reducing environment of subcellular organelles has been exploited mainly by incorporating a disulfide bond(s) into the structure of the delivery vectors to provide them with a capability to release the therapeutic nucleotides selectively in the subcellular reducing space. The original interest in gene delivery systems controlled by redox potential gradients was guided by the need to transiently enhance stability of the vectors during the delivery [Cheng et al., 2011; Meng et al., 2009].

The design of reduction-sensitive polymers and conjugates usually involves incorporation of disulfide linkage(s) in the main chain, at the side chain or the cross-linker in the structure of the polymers of either linear or branched structure. There are only a few examples of polyplexes where the disulfide bonds are associated with the nucleic acids [Soundara Manickam and Oupický, 2006]. Reduction-sensitive polymers and conjugates are characterized by an excellent stability in the circulation and in extracellular fluids, whereas they are prone to rapid degradation under a reductive environment present in intracellular compartments such as the cytoplasm and the cell nucleus at a time scale from minutes to hours, through thiol-disulfide exchange reactions. This quick-response chemical degradation behavior is distinct from common hydrolytically degradable polymers such as aliphatic polyesters and polycarbonates in which the ester and carbonate bonds usually exhibit gradual degradation kinetics inside body with degradation times ranging from days to weeks/or to months. This remarkable feature renders them extremely for the controlled cytoplasmic delivery of a variety of bioactive molecules including DNA, siRNA, antisense oligonucleotide (ODN), proteins, drugs, etc [Du et al., 2010].

Disulfide bonds present in the structure of polyplexes are readily reduced in the reducing intracellular environment, while largely preserved in the predominantly oxidizing extracellular 
space. The intracellular reduction of disulfide bonds is most likely mediated by small redox molecules like glutathione (GSH) and thioredoxin, either alone or with the help of redox enzymes. Glutathione tripeptide (g-glutamyl-cysteinyl-glycine; GSH) is the most abundant intracellular sulfhydryl present in millimolar concentrations inside the cell but only in micromolar concentrations in the blood plasma and GSH/glutathione disulfide (GSSG) is the major redox couple in animal cells [Meng et al., 2009]. Glutathione has multiple direct and indirect functions in many critical cellular processes like synthesis of proteins and DNA, amino acid transport, enzyme activity, metabolism and protection of cells. Glutathione also serves as a reductant by functioning to destroy the free radicals, hydrogen peroxide and other peroxides. It also functions as a storage form of cysteine. The intracellular glutathione concentration is an additive function of both the oxidized (GSSG) and the reduced forms (GSH) of glutathione. The glutathione redox ratio is maintained and determined by the activity of glutathione reductase, NADPH concentrations and transaldolase activity. The redox state of the GSH/GSSG couple is often used as an indicator of the overall redox environment of the cell. The large difference in reducing potential between the intracellular and extracellular milieu may be exploited for triggered intracellular delivery of a variety of bioactive molecules including DNA, siRNA, antisense oligonucleotide (ODN), proteins and low molecular weight drugs. Furthermore, of particular interest is that tumor tissues are highly reducing and hypoxic compared with normal tissues, with at least 4-fold higher concentrations of GSH in the tumor tissues over normal tissues, rendering the reducible bioconjugates valuable for tumor-specific drug and gene delivery [Meng et al., 2009]. Some examples are mentioned for the use of redox responsivenanocarrier in below:

\subsubsection{The use of responsive sensitive nanocarrier for the stabilization of the carrier and decrease the cytotoxicity}

A prerequisite for every systemic nucleic-acid delivery system is stability in the blood stream prior to reaching its target cell. Therefore, the carrier must prevent the premature release of its load. In order to enhance the stability of the delivery system, either the surface of the carriers was crosslinked, or the low-molecular-weight materials were sulfhydryl polymerized. Peptides containing many lysines and histidines were used for the complexation of nucleic acids, while cysteines were introduced to obtain stable and reductively degradable carriers. A series of different vehicles was built from sequences of many lysines, tryptophan, and a variable number of cysteines. All peptides obtained were able to condense DNA into particles, and exhibited increased stability after disulfide formation. The disulfide crosslinked carriers conveyed 5- to 60 -fold higher gene expression as compared to their non-crosslinked analogs. The degree of gene expression was dependent on the number of incorporated cysteines, with a maximum for terminally inserted cysteines. The modification of the e-amino groups of pLL with 3-(2-aminoethyldithio) propionyl residues or their crosslinking with Dimethyl 3,3-dithiobispropionimidate (DTBP) comprises another approach utilizing reductively degradable gene carriers. These molecules were able to complex nucleotides into particles that were stable under physiological conditions but disintegrated upon treatment with GSH [Meng et al., 2009].

Read et al. prepared gene delivery vectors based on reducible polycations (RPCs) by oxidative polycondensation of the peptide Cys-Lys10-Cys and used to condense nucleic acids. The 
release of nucleic acids in these vectors relied on cleavage of the RPC in the reduced intracellular environment, eliminating the toxicity associated with high molecular weight polymers. However these polymers rely on chloroquine or cationic lipids to enhance endosomal escape and mediate transfection [Read et al., 2003].

Low molecular weight DNA condensing polypeptides were developed by substituting one to four lysine residues of Cys-Trp-Lys18 (CWK18) with cysteine groups. These polypeptides could spontaneously oxidize to form interpeptide disulfide crosslinks after binding to plasmid DNA, resulting in small stabilized DNA condensates. These reversibly cross-linked polypeptide DNA condensates were 5-60-fold more potent at mediating gene expression in HepG2 and COS-7 cells as compared to the un-crosslinked alkylated CWK18 (AlkCWK18) DNA condensates [Fig. 2 A; McKenzie et al., 2000]. In another study, in order to improve the endosomolytic properties, Histidine containing reducible polycations based on $\mathrm{CH} 6 \mathrm{~K} 3 \mathrm{H} 6 \mathrm{C}$ monomers (His6 RPCs) was developed, which are highly effective DNA transfection agents, provided sufficient buffering capacity and enhanced in vitro gene expression with rapid unpackaging following reduction in the cytoplasm without aid of chloroquine, an agent that promotes endosomal escape [Stevenson et al., 2008].

High molecular weight polypeptides containing disulfide bonds in the backbone were synthesized by an oxidative copolymerization of a histidine-rich peptide (HRP) and a nuclear localization sequence (NLS) peptide derived from the importin $\alpha$-binding SV40 T antigen sequence. The synthetic approach allowed an easy synthesis of reducible copolypeptides (rCPP) with different relative contents of the HRP and NLS sequences. The rCPPs were synthesized by DMSO-mediated oxidative polycondensation. To combine two functional peptides into a single polymeric carrier, multiblock reducible copolypeptides were synthesized by randomly connecting HRP and NLS peptides via disulfide bonds into a linear polypeptide chain. Mild oxidation of the terminal Cys residues with DMSO was used. The copolypeptides show minimal cytotoxicity and transfection activity comparable to or better than control PEI polyplexes [Manickam and Oupický, 2006].

Other carriers, such as chitosan (deacetylated chitin) were also stabilized via disulfide bonds. For this purpose, the primary amino groups of low-molecular-weight chitosan were thiolated with 2-iminothiolane. These modified polymers, such as chitosanthiobutylamidines, were mixed with pDNA to form coacervates in the nanometer range [Bauhuber et al., 2009]. In a recent study, Ho et al. modified chitosan (CS) with extending arms consisting of disulfide spacers and arginine (Arg) residues (CS-SS-Arg) as a novel non-viral carrier for gene delivery. Cleavage of disulfide spacers by glutathione (GSH) and dithiothreitol due to thiol-disulfide exchange reactions indicates that CS-SS-Arg is likely reducible in cytoplasm. The CS-SS-Arg was allowed to condense GFP DNA to form self-organized nanoparticles with a diameter of $130 \mathrm{~nm}$ and zeta potential of $35 \mathrm{mV}$. The DNA was released from CS-SS-Arg/ DNA nanoparticles over time in the presence of GSH and the results suggest that the Arg-rich bioreducible CS-SS-Arg/ DNA nanoparticles are promising as a carrier for gene delivery [Ho et al., 2011].

A dilemma in non-viral nucleic acid delivery is demonstrated by considering the polymeric transfection agent PEI, which is often referred to as the gold standard for polymer-based 
gene carriers due to the relatively high transfection efficacy of its polyplexes. Unfortunately, efficacy and adverse reactions seem to be strongly associated with the use of PEI. A popular strategy to reduce the toxicity of polyplexes is to use low MW polycations or cationic monomers that are less cytolytic, and crosslink them with agents that can be cleaved or activated by the intracellular environment. Numerous reports describe the synthesis of carrier systems containing disulfide bonds that allow for a compaction and protection of the nucleic acid in the extracellular environment accompanied by reduced toxicity due to intracellular polymer degradation. Peng et al. prepared thiolated PEI (800 Da) to further oxidize into disulfide cross-linked PEI (PEI-SS), with average molecular weights of 7.1, 8.0 and $8.4 \mathrm{kDa}$ depending on the degree of thiolation. Those PEI-SS had lower cytotoxicities and higher transgene expressions compared with that of the 25-kDa branched PEI (bPEI) [Peng et al., 2008].

(A)
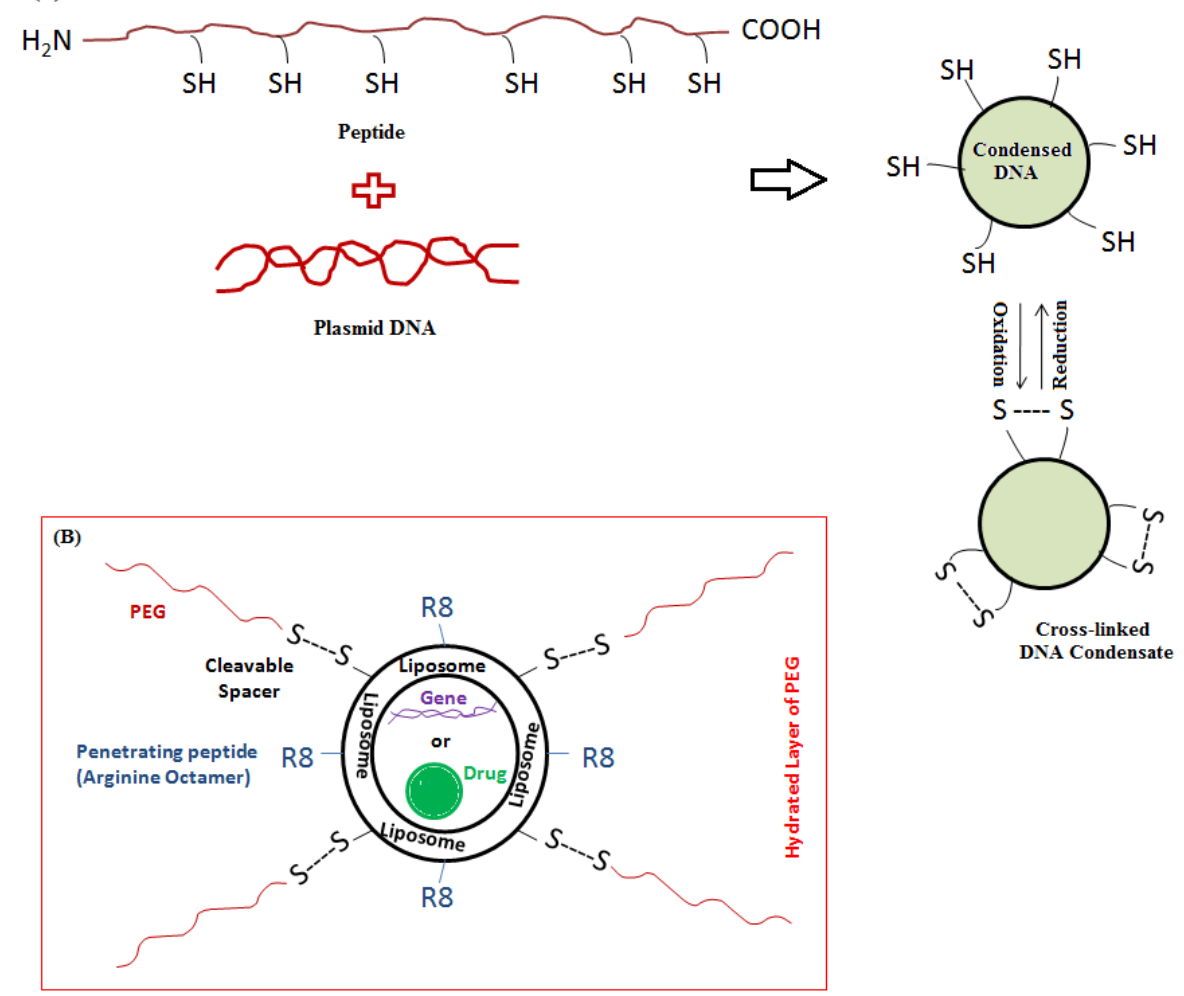

Figure 2. A) Cross-linked peptide-DNA condensates: Formation of peptide-DNA condensates through ionic binding of the peptide to the pDNA followed by interpeptide oxidation; Stabilization of the DNA condensates by reversible disulfide bonds. B) A novel stimuli-sensitive liposome: This carrier composes of cationic peptides (e.g., Arginine octamer) and detachable coat (e.g., PEG) for the intracellular gene or drug delivery 
Kang et al. synthesized reducible polycations (RPC) that degrade due to changes in the intracellular reduction potential from low molecular weight (MW) bPEI $0.8 \mathrm{kDa}$ via thiolation and oxidation. In this study, 2-iminothiolane was used to create thiol groups from primary amines. In this approach, a primary amine reacts with 2-iminothiolane to yield a thiol group and an amidine moiety. A procedure based on the use of 2-iminothiolane was employed in order to avoid problems associated with the use of DTBP. As a result, unlike other thiolation methods, by converting primary amines to amidine groups, the number of positive charges in the final product at a neutral $\mathrm{pH}$ was preserved. Moreover, the newly generated amidines contribute to the electrostatic condensation of nucleic acids. The cytotoxicity of RPC-bPEI $0.8 \mathrm{kDa}$ was 8-19 times less than that of the gold standard of polymeric transfection reagents, bPEI $25 \mathrm{kDa}$. In general, the toxicity of High MW (HMW) PEI is greater than that of Low MW (LMW) PEI because HMW PEI interacts more effectively with components that are essential for cell survival such as intracellular membranes, vital proteins, and nucleic acids than its LMW counterpart. Thus, the reduced cytotoxicity of HMWRPC-bPEI $0.8 \mathrm{kDa}$ may be due to the degradation of the polymer in the intracellular environment after cellular uptake [Kang et al., 2011].

In another study, Son et al developed a multi-functional gene carrier based on thiolated low molecular weight bPEI with functional moieties for cytoplasm-sensitive reduction, tumor targeting, and prolonged circulation in blood. The bPEI was modified with $\alpha$-maleimide- $\omega$ N-hydroxysuccinimide ester polyethylene glycol (MAL-PEG-NHS, MW: 5000) and cyclic NGR peptide for enhanced blood compatibility and tumor targeting ability. The resulting polymer (bPEI-SS-PEG-cNGR) exhibited DNA condensing capacity, a reducing property, and improved tumor targeting, suggesting that the multifunctional polymer constitutes a promising non-viral vector for cytoplasm- and tumor-specificities [Son et al., 2010].

\subsubsection{Disulfides for the attachment of a shielding moiety}

DOPE and N-[2-methoxypoly (ethylene glycol)- $\alpha$-aminocarbonylethyl-dithiopropionate] formed a liposome that was covalently coupled to distearoylphosphatidylethanolamine (mPEG-SS-DSPE). The incorporation of the mPEG-SS-DSPE stabilized these liposomes at low $\mathrm{pH}$, but the stabilizing effect was quickly reversed when the carrier was incubated with either DTT or cell-free extracts. Under these conditions, the disulfides were reduced, and thus the protecting PEG chains were cleaved, and the vehicle became degradable at low $\mathrm{pH}$, releasing its load. Disulfides were used for the formation of copolymers consisting of bPEI $25 \mathrm{kDa}$ and PEG of 20 or $30 \mathrm{kDa}$. The connection of PEG via DSPE led to a 125\% increase in blood levels and decreased hemolysis compared to bPEI $25 \mathrm{kDa}$ [Bauhuber et al., 2009].

Polyion complex (PIC) micelles are self-assembling particles with a core-shell structure formed by complexation between a pair of oppositely charged polymers having hydrophilic PEG segments. They can be utilized as gene delivery vectors with high water-solubility and colloidal stability, employing negatively charged genetic materials and cationic polymers. One approach to enhance the stability of PIC micelles is glutathione (GSH)-sensitive stabilization of PIC micelles with the core cross-linked through disulfide bonds, composed of antisense oligonucleotide and thiolated PEG-PLL block copolymer. The micelles showed 
sufficient colloidal stability due to the PEG shell and core cross-linking. Moreover, ODN entrapped in the micelles also displayed highly increased stability against nuclease, compared to that in the micelles without cross-linking. Release of ODN from the dissociated micelles at intracellular GSH concentration suggested the potential for intracellular ODN delivery. It is reported that PEG-peptide (PEG-SS-Cys-Trp-Lys(18): PEG-SS-CWK18) having disulfide cross-linker displayed much enhanced gene transfer efficiency compared to PEGpeptide having non-reducible cross-linker (PEG-VS-CWK18) when complexed with plasmid DNA [Kim and Kim, 2011].

\subsubsection{Disulfides for the attachment of a targeting moiety}

For a successful accumulation of the nucleic acid in the target cell, the delivery system requires the attachment of a specific "recognition element". However, since many targeting moieties are rather large, they hinder effective unpacking of the gene vector. Thus, inside cells, it is very important to remove them to facilitate the disassembly of the load. Here, disulfides can be quite useful, as they can be cleaved at the cell surface during cellular entry or in the cytosol [Bauhuber et al., 2009]. The frequency of sulfhydryl occurrence in Antibodies/proteins or other molecules is usually low as compared to other groups like amines or carboxylates. The use of sulfhydryl reactive chemistries thus can restrict modification to only a limited number of sites within a target molecule. N-Succinimidyl 3-(2-pyridyldithio) propionate (SPDP) is one of the most popular heterobi-functional cross-linking agents. The NHS ester end of SPDP reacts with amine groups to form an amide linkage, while the 2-pyridyldithiol group at the other end can react with sulfhydryl residues to form a disulfide linkage. The reagent is also useful in creating sulfhydryls in proteins and other molecules. Once modified with SPDP, a protein can be treated with DTT (or other disulfide reducing agents, to release the pyridine-2-thione leaving group and form the free sulfhydryl [Manjappa et al., 2011].

Iminothiolane (Traut's reagent) can react with primary amines in a ring-opening reaction that regenerates the free sulfhydryl. An example is the thiolation of antibody using Traut's reagent in the preparation of immunoliposomes. It is an excellent thiolation reagent for use in the preparation of immunotoxins. It has also been used to modify and introduce sulfhydryls into oligosaccharides from asparagines linked glycans [Manjappa et al., 2011].

\subsubsection{Disulfide bonds to enhance the intracellular release of the nucleic acid}

SiRNA has generated great interest as a research tool and a therapeutic agent because of its ability to efficiently silence specific genes by the mechanism of RNA interference [Vidugirienè et al., 2007]. However, siRNA cannot penetrate the cellular membrane alone and can be easily degraded by RNase; therefore, effective siRNA carriers are needed for siRNA-based therapies. In this regard, poly ion complexes (PICs) formed from nucleic acids and oppositely charged polycations have been widely studied as a promising nucleic acids carrier because of the variety of chemical designs of polycations. PICs protect nucleic acids from enzymatic degradation and show facilitated cellular uptake. Thus, PICs have demonstrated to be useful for plasmid DNA (pDNA) delivery in vitro and in vivo. 
Nevertheless, there has been limited success to date in the development of PICs for siRNA delivery. In general, PICs from monomeric siRNA, which has a short structure compared to pDNA, lack stability under the physiological condition; therefore, substantial stabilization of PICs is essential to successful siRNA delivery. Meanwhile, after PICs internalize and reach the cytoplasm, they are required to efficiently release siRNA to exert its gene silencing effect. To complete such variable properties, considerable efforts have been dedicated to the design and chemical modification of polycations [Yu-Lin et al., 2011]. Takemoto et al. reported a new class of chemically modified siRNA, i.e., siRNA-grafted poly (aspartic acid) [PAsp (-SS-siRNA)], for PIC based siRNA delivery. PAsp (-SS-siRNA) consists of a backbone of a poly (aspartic acid) [PAsp] derivative and grafted siRNAs via a disulfide linkage. The siRNA-grafted polymer formed stable PICs due to its larger numbers and higher density of anionic charges compared with monomeric siRNA, leading to effective internalization by cultured cells. Following the endosomal escape of the PIC, the disulfide linkage of the siRNA-grafted polymer allowed efficient siRNA release from the PIC under intracellular reductive conditions. Consequently, the PIC from the siRNA-grafted polymer showed a potent gene silencing effect without cytotoxicity or immunogenicity, demonstrating a promising feature of the siRNA-grafted polymer to construct the PICbased nanocarrier for in vivo siRNA delivery [Takemoto et al., 2010].

The conjugation of nucleic acids including siRNA and antisense oligodeoxynucleotide to polymer such as PEG and hyaluronic acid through a disulfide bond represents a approach to construct GSH-responsive gene delivery systems [Cheng et al., 2011]. Lee et al. explored the potential possibility of hyaluronic acid (HA) as a biocompatible, biodegradable, and non-cytotoxic material for delivery of siRNA. Nano-sized HA hydrogels, called HA nanogels, were prepared for target-specific intracellular delivery of siRNA to HA receptor over-expressing cancer cells. HA nanogels crosslinked with disulfide linkages were prepared by an inverse emulsion method. An aqueous phase containing thiol functionalized HA and siRNA was emulsified in an oil phase under ultrasonication, thus generating self cross-linked HA nanogels encapsulating anti-green fluorescent protein (GFP) siRNA. Release profiles of siRNA from HA nanogels were studied in response to various reductive conditions that could cleave the disulfide linkages of HA nanogels to varying extents. The HA/siRNA nanogels were readily taken up by HA receptor positive cells (HCT-116 cells) having HA-specific CD44 receptors on the surface [Lee et al., 2007]. Kam et al. conjugated various biological molecules, including oligonucleotides and siRNA, to phospholipid-PEG functionalized single-walled carbon nanotube (SWNT) via cleavable disulfide linkage. Due to the presence of a PEG linker, these SWNT conjugates form highly stable suspensions in aqueous solutions including physiological buffers. The SWNT carriers mediated efficient delivery and release of DNA and siRNA inside cells. Gene silencing experiments using HeLa cells displayed a two-fold higher silencing efficiency compared to lipofectamine at the same siRNA concentration. This is ascribed to a high surface area of SWNT for efficient siRNA cargo loading, high intracellular transporting ability of SWNT, and high degree of endosome/lysosome escape owing to the disulfide approach [Kam et al., 2005; Meng et al., 2009]. 


\section{Stimuli-sensitive multi-functional nano-particulate nucleic acid delivery systems}

As mentioned in the previous sections, there are some conflicting demands for overcoming different extra- and intracellular barriers met by nucleic acid-loaded nano-particles during delivery. The delivery systems, which response to only one stimulus and have less functionality, may not be efficient enough to achieve a satisfactory therapeutic effect in vivo. Natural gene carriers, such as viruses, have developed sophisticated mechanisms and modular biopolymer designs to overcome these barriers. The development of virus-mimicking, multifunctional gene delivery systems with features that mimic virus modular components and which transfect specific cell lines with high efficacy is considered to be a practical strategy in the future, in particular for intravenous administration. Ideal polymer-based, nucleic acidloaded nanoparticles for potential in vivo applications should have several components that function at the appropriate stages during the delivery. The hierarchical nature of the synthetic carriers allows the incorporation of membrane-disrupting peptides, nucleic acid binding components, a protective coat layer, and an outer targeting ligand all in a single nanoparticle, but with functionality such that each is utilized in a specific sequence during the gene delivery process [Du et al., 2010].

The ligands on the particle surface can be used to recognize a specific cell/tissue, and facilitate cellular uptake through receptor-mediated endocytosis. A reversibly removable hydrophilic pole, such as PEG chains, provides stealth protection of the nanoparticles during their circulation in the blood as well as travelling through the ECM, and may reduce toxicity. Moieties that are responsive to different stimulus must be combined to result in an appropriate set of various trigger mechanisms at different time points. Such moieties might be cleaved in acidic environments, show good buffering capacity, be redox active or enzymatically cleavable, the pole can be removed, which might enhance the cellular uptake and/or endosomal escape. In the inner part of the nanoparticles, nucleic acids can be temporally loaded through electrostatic interaction, covalent conjugation, or physical encapsulation, which will protect the nucleic acid against enzymatic degradation. The inner part should be stable enough until delivery to the correct site (cytoplasm and/or nucleus) where it is disassembled to release the naked nucleic acid upon some kind of stimulus. In addition, it is preferred that the inner part contains endosomolytic components that help the endosomal escape. If cell penetrating peptide (CPP) is incorporated onto the surface of the inner part, cellular uptake may be further improved [Du et al., 2010]. Many experimental results emphasize that the main obstruction of CPPs is the absence of specific cellular delivery [Vivès et al., 2008]. Ideally, the design of a smart delivery system should be built in such a way that during the first phase of delivery, a non-specific cell-penetrating function is shielded by the function of cell-recognition motif which favors the concentration of the drug at the targeted cell type. Upon accumulating in the target, protecting polymer (or specific ligand) attached to the surface of the smart nanocarrier via the stimuli-sensitive bond should detach under the action of local conditions and expose the previously hidden second function (CPP) allowing for the subsequent delivery of the carrier and its cargo inside cells [Torchilin, 2008]. Such engineering of smart nanodevices has 
been illustrated in the work by Sawant et al. who developed targeted long-circulating PEGylated liposomes and PEG-phosphatidylethanolamine (PEG-PE)-based micelles possessing several functionalities. Such systems were capable of targeting a specific cell or organ by attaching the monoclonal antibody to their surface via long PEG spacer groups. Second, these liposomes and micelles were additionally modified with TATp moieties attached to the surface of the nanocarrier by using TATp-short PEG-PE derivatives. PEG-PE used for liposome surface modification or for micelle preparation was made degradable by inserting the $\mathrm{pH}$-sensitive hydrazone bond between PEG and PE (PEG-Hz-PE). Under normal pH values, TATp functions on the surface of nanocarriers were "shielded" by long protecting PEG chains (pH-degradable PEG 2000-PE or PEG 5000-PE) or by long para- nitrophenyl PEG-PE (pNP-PEG-PE) moieties used to attach antibodies to the nanocarrier (non-pH degradable PEG3400-PE or PEG5000-PE). Following prolonged circulation and uptake into the tumor mass mediated by both the enhanced permeability and retention (EPR) effect and the active targeting agent on the surface of the carrier, the $\mathrm{pH}$ responsive bonds are cleaved in the acidic environment of the tumor, thereby releasing the high molecular weight PEG strands from the carrier and exposing the Tat-peptide for enhanced intracellular uptake and intracellular target localization, specifically located to the target tumor cells [Sawant et al., 2006; Torchilin, 2008].

Another example of a triggerable system was created by the synthesis of a liposomal carrier that was comprised of a membrane-permeable ligand and a reductively detachable PEG coating. The surface cover consisted of PEG coupled to DOPE via a thiolytically cleavable linker, while the ligand, an ariginine octamer, was immobilized onto cholesteryl hemisuccinate (CHEMS). These conjugates were mixed with dipalmitoylphosphatidylcholin (DPPC) and unmodified DOPE to form liposomes. DOPE/mPEG-DTP-DSPE liposomes were stable in plasma whereas in the presence of a reducing agent the PEG coating was effectively detached off the liposomal surface, leading to vesicle destabilization and fusion as well as complete release of the entrapped contents. Upon activation of the trigger, the arginine octamer would be exposed, causing cellular uptake of the liposome. Once in the cytosol, the remaining disulfide bonds would be cleaved, ultimately resulting in the destabilization the whole carrier [Fig. 2B; Bauhuber et al., 2009].

\section{Nanoparticles mediated brain delivery systems}

Nanoparticles have emerged as potential drug delivery carriers to tissues throughout the body. Yet passing the BBB is particularly difficult. The proper design of such engineered 'nanocarriers' becomes very important in transversing the impermeable membranes to facilitate drug delivery. At the same time, it is also required to retain the drug stability and ensure that early degradation of drugs from the nanocarriers does not take place. Therefore, for drugs to be successfully delivered to their target, many factors such as its size, biocompatibility, target specific affinity, avoidance of reticuloendothelial systems, stability in blood, or ability to facilitate controlled drug release need to be considered during manufacture of the NPs. As for nanocarriers to serve as good candidates for drug delivery across the BBB can be summarized as following [Bhaskar et al., 2010]: a) Particle diameter less than 100 nanometers; b) Non-toxic, 
biodegradable and biocompatible; c) Stable in blood (i.e., no opsonisation by proteins); d) BBBtargeted (i.e., use of cell surface, ligands, and receptor mediated endocytosis); e) No activation of neutrophils, non-inflammatory; f) No platelet aggregation; h) Avoidance of the reticuloendothelial systems; i) Prolonged circulation time; j) Scalable and cost effective with regard to manufacturing process; m) Agreeable to small molecules, peptides, proteins or nucleic acids; n) Controlled drug release or modulation of drug release profiles [Bhaskar et al., 2010].

One of the most important challenges in nano-based diagnostics and drug delivery is the functionalization of nanoparticles. At first, the combination of effective conjugation strategies is needed to develop, in a highly controlled way, specific biomolecules to the surface of nanoparticles. Some of the most prominent candidate biomolecules are cell penetrating peptides such as SynB vectors, penetratin and Tat that facilitate enhanced intracellular delivery, fluorescent dyes (rhodamine, alexa, Cy5.5), tumoral markers for brain and gene therapeutic agents for genetic therapy such as siRNA [Bhaskar et al., 2010].

Functionalization itself requires a profound knowledge of the target organ and its transport mechanisms. The BBB has several transport molecules that can potentially increase the efficiency and kinetics of nanocarriers towards brains such as, growth factors (e.g. epidermal growth factor, vascular endothelial growth factor, basic fibroblast growth factor, insulin-like growth factors (IGF-I and -II), biotin binding proteins (avidin, streptavidin, or neutravidin), insulin, albumin, leptin, lactoferrin, iron binding protein p97 (melanotransferrin), transferrin and Angiopep-2. Some agents play a pivotal role in enhancing the permeability of nanoprobes through BBB. Moreover, by altering the surface of polymeric nanoparticles on coating them with different hydrophilic surfactants, such as polysorbate 80 (Tween ${ }^{\circledR} 80$ ) or other polysorbates with 20 polyoxyethylene units, biocompatible coatings of non-viral gene delivery systems, e.g. by PEG attachment for siRNA delivery show significant advantage in brain targeting [Bhaskar et al., 2010].

\section{Cell penetrating peptides as efficient delivery systems}

Generally, the nucleic acid delivery techniques comprise various physical and chemical methods, viral and non-viral vector systems, and uptake of naked nucleic acids [Veldhoen et al., 2008; Bolhassani et al., 2011]. All of them have certain advantages and dis-advantages and might only be appropriate if particular requirements are performed. For instance, physical and chemical methods like microinjection, electroporation or particle bombardment as well as calcium phosphate co-precipitation are highly efficient but rather harmful for the target cells and lack the potential to be applicable in vivo. There is general agreement that viral vector systems are the most efficient vehicles to deliver nucleic acids into cells [Veldhoen et al., 2008]. However, despite substantial efforts over the last 15 years, up to now research has failed to develop suitable and especially safe viral systems. As a result of the difficulties encountered with these viral vectors (e.g., mutagenesis and immune responses), much attention was paid to the development of safer non-viral delivery systems. Currently, liposomes and cationic polymers are used as a standard tool to transfect cells in vitro. These approaches are yet 
characterized by a significant lack of efficiency accompanied by a high level of toxicity making them mostly inadequate for in vivo applications [Veldhoen et al., 2008; Bolhassani et al., 2011].

Peptides acting as shuttles for a controlled cellular delivery of nucleic acids represent a new concept to bypass the problem of poor bio-availability and clinical efficacy of such macromolecules [Veldhoen et al., 2008]. The idea of using peptides as carriers goes back some 20 years when two groups discovered by chance that the HIV-1 transactivating protein Tat is taken up by mammalian cells [Frankel and Pabo, 1998; Green and Loewenstein, 1988]. Just a few years later, the Antennapedia homeodomain of Drosophila melanogaster was shown to act similarly [Joliot et al., 1991]. Then, it could be shown that peptides derived from Tat and Antennapedia as well as other proteins are capable of transporting macromolecular cargo molecules into cells [Fawell et al., 1994; Schwarze et al., 1999]. Based on such promising results, a rapidly expanding field focusing on the so-called cell-penetrating peptides (CPPs), also referred to as protein transduction domains (PTD) began to develop [Veldhoen et al., 2008].

Up to now numerous CPPs have been described. According to their origin, they can be grouped into three classes. The first group contains CPPs originating from naturally occurring proteins "protein derived CPPs", the second consists of chimeric CPPs composed of different protein domains and the third class includes so-called "model CPPs" which were developed according to structure-fuction relationships without any homology to natural sequences. All known CPPs are basic amino acids causing a net positive charge at physiological $\mathrm{pH}$ [Langel, 2002].

At present, a peptide is considered a CPP, if it shows the ability to cross a biological membrane. A cargo can be bound to the CPP covalently or non-covalently. Covalent attachment can be achieved either by expression as a fusion construct or by chemical coupling [Zatsepin et al., 2005]. In some cases, cargo and carrier bind each other non-covalently through ionic interactions. Depending on the nature of both binding partners assembly of nanoparticles may occur [Veldhoen et al., 2008].

The studies have shown that the cargo-CPP complexes are taken up by directly penetrating the cell membrane or by an endocytotic pathway. However, the precise mechanism of internalization remains elusive and strongly depends on the properties of both CPP and cargo as well as the transfection conditions and the cell lines used [Maiolo et al., 2005; De Coupade et al., 2005; El-Andaloussi et al., 2007; Bolhassani, 2011]. Recent studies indicate that the uptake mechanism of CPPs can be influenced by the attachment of cargo. For example, Richard et al. [Richard et al., 2003; Richard et al., 2005] reported a co-localization of Tat ${ }^{48-59}$ with markers of clathrin-mediated endocytosis, whereas Fittipaldi et al. [Fittipaldi et al., 2003] found a caveolae/ lipid raft-dependent process for a Tat-GFP fusion protein and Wadia et al. [Wadia et al., 2004] described a macropinocytotic uptake pathway for a fusion construct of Tat peptide with Cre recombinase.

However, further information about the exact mechanism of uptake of such delivery systems is expected in the near future. Furthermore, it has been shown that even minor changes of the physical state of a CPP (e.g., exchange of certain amino acids) can alter translocation properties significantly. This particularly holds true for the attachment of large cargo molecules [Veldhoen et al., 2008]. Thus, it might not be possible to generalize results obtained with a CPP, 
and it might be necessary to characterize each carrier/cargo complex individually. If CPPs are proposed to be used for therapeutic purposes in the future, it is essential to focus on the attachment of functional cargos and analyze their biological effects inside the cell. Data from our lab clearly show that uptake and biological activity of a functional cargo is everything but the same. Therefore, a quantitative comparison of cargo taken up and functionally active cargo is an essential requirement in order to improve therapeutic efficacy. Indeed, only looking for efficient internalization is not sufficient [Veldhoen et al., 2008].

The successful clinical application of nucleic acid-based therapeutic strategies has been limited by the poor delivery efficiency achieved by existing vectors. The development of alternative delivery systems for improved biological activity is, therefore, obligatory. Since two decades ago that the Tat protein, and derived peptides, can translocate across biological membranes, cell-penetrating peptides have been considered one of the most promising tools to improve non-invasive cellular delivery of therapeutic molecules. Despite extensive research on the use of CPPs for this purpose, the exact mechanisms underlying their cellular uptake and that of peptide conjugates remain controversial [Trabulo et al., 2010].

There are many examples of CPP-mediated delivery of plasmid DNA into cultured cells and also in vivo involving the use of a non-covalent approach [Bolhassani, 2011]. While some approaches involve single component peptide vectors, the major focus has been on the association of CPPs with other non-viral gene delivery methods, such as liposomes, polyethyleneimine (PEI) or nanoparticles. In 1999, Morris and coworkers demonstrated that MPG could be used as a powerful tool for the delivery of nucleic acids. It was shown that MPG is not cytotoxic, insensitive to serum and able to efficiently deliver plasmid DNA into several different cell lines [Morris et al., 1999]. Further studies demonstrated that cell entry of the MPG/ DNA particles is independent of the endosomal pathway and that the NLS of MPG is involved in both electrostatic interactions with DNA and nuclear targeting. Furthermore, it was shown that a mutation affecting the NLS of MPG prevents nuclear delivery of DNA. In an alternative study, Rittner et al. described the novel basic amphiphilic peptides, ppTG1 and ppTG20 (20 amino acids), and evaluated their efficiencies in vitro and in vivo as single-component gene transfer vectors. It was demonstrated that both the ppTG1 and ppTG20 peptides are able to bind nucleic acids and destabilize membranes, in a liposome leakage assay. Complexes of plasmid DNA with ppTG1 originated high levels of gene expression in cell culture experiments and, most importantly, complexes of plasmid DNA with ppTG1 or ppTG20 led to significant gene expression in vivo [Rittner et al., 2002]. Peptide modification has also been explored as a means to enhance gene delivery. In particular, stearic acid modification of different membranepermeable arginine-rich peptides, such as HIV-1 Tat (48-60), HIV-1 Rev (34-50), flock house virus (FHV) coat (35-49), (RxR)4 and oligoarginines of 4-16 residues was shown to substantially increase their transfection efficiency. The mechanisms by which stearic acid modification improves plasmid DNA delivery by CPPs have been shown to involve increased efficiency of endosomal escape or enhanced cellular association, as well as higher nuclear delivery. The extensively studied Tat peptide has also been exploited for plasmid DNA delivery by different research groups, with paradoxical results [Trabulo et al., 2010, Bolhassani, 2011]. A study by Ignatovich et al., demonstrated that Tat peptide is able to form complexes with plasmid DNA, 
which could be used for gene delivery into mammalian cells. Despite reasonably high transfection efficiency in vitro, low gene expression levels were detected in the liver of mice injected intravenously with DNA-Tat complexes, a fact that was attributed to inactivation of the complexes in the bloodstream due to interactions with serum albumin. Interestingly, an endocytosis-dependent mechanism was proposed for the uptake of the DNA-Tat complexes, similar to what was proposed for internalization of complexes of plasmid DNA with other polycationic carriers. A different study, by Tung et al., compared the efficiency of a series of Tat peptides, containing 1-8 Tat moieties. Although, all compounds complexed with plasmid DNA, it was demonstrated that at least eight Tat peptide moieties are required in order to achieve efficient gene delivery. Sandgren et al. also studied the cellular uptake of complexes of plasmid DNA and the HIV-Tat derived peptide. According to this study, the Tat peptide stimulated cellular uptake of DNA in a time-, concentration- and temperature-dependent manner, while accumulating in large, acidic, cytoplasmic vesicles, followed by transfer of the cargo into the nuclear compartment and subsequent disappearance from the endolysosomal vesicles. Aiming at increasing the efficiency of the Tat peptide to deliver plasmid DNA, Lo et al. made several modifications to the Tat peptide, through the use of histidine and cysteine residues to enhance endosomal escape and complex stability [Trabulo et al., 2010]. Up to 7,000fold improvement in gene transfection efficiency was observed for the Tat peptide covalently fused with 10 histidine residues (Tat- $10 \mathrm{H})$ over the original Tat peptide, and incorporation of two cysteine residues into this peptide resulted in an even higher efficacy (C-5H-Tat-5H-C). The association of CPPs with other non-viral delivery vectors has also been extensively investigated, aiming at exploring the possibility to combine efficient delivery, packaging and targeting moieties within the same system [Trabulo et al., 2010].

A combination of a peptide nucleic acids (PNA) with the SV40 core NLS, performed by Branden et al., originated a bifunctional peptide that improved the efficacy of plasmid transfection up to 8-fold when associated with the transfection agent polyethyleneimine (PEI) [Branden et al., 1999]. Several other studies also combined PEI with CPPs. Kleemann et al. covalently coupled the Tat peptide to $25 \mathrm{kDa}$ PEI through a heterobi-functional PEG spacer resulting in a Tat-PEG-PEI conjugate. Improved DNA reporter gene complexation and protection were observed for small (approximately $90 \mathrm{~nm}$ ) polyplexes as well as low toxicity and significantly enhanced transfection efficiency in vivo [Kleemann et al., 2005]. Rudolph et al. demonstrated that oligomers of the Tat peptide were able to condense plasmid DNA to nanosized particles and protect DNA from nuclease degradation [Rudolph et al., 2003]. Most importantly, when DNA was pre-condensed with Tat peptides and PEI, Superfect or LipofectAMINE were added to the mixture, transfection efficiency was enhanced up to 390-fold compared with the standard vectors. Similar studies by Kilk et al., demonstrated that the poor transfection abilities exhibited by TP10 was significantly enhanced in the presence of PEI, increasing several fold compared to PEI alone, particularly at low PEI concentrations, therefore allowing the use of reduced PEI concentration [Kilk et al., 2005]. Using fluorescently labeled liposomes and cargos, Torchilin et al. demonstrated that large drug carriers, such as $200 \mathrm{~nm}$ liposomes, could be delivered into cells by attaching Tat peptide to the liposome surface. Next, the same group described the formation of non-covalent complexes of Tat, liposomes and DNA that were able to efficiently transfect cells both in vitro and in vivo, while being less toxic than 
other commonly used transfection reagents. The internalization of this system was claimed to depend on a direct cytoplasmic delivery imparted by the Tat peptide [Torchilin et al., 2001]. A study by Hyndman et al. showed that mixing the Tat with liposomes containing DOTAP or Lipofectin and DNA, resulted in complexes that significantly enhance transfection in vitro with a marked reduction in the amount of liposomes required, despite the lack of any covalent linkage of the peptide to liposomes. In this study, the use of endosomolytic agents and results from experiments performed at low temperature suggested that the endocytotic pathway was involved in the internalization of the complexes. Another report demonstrated that the increase in gene transfer of Tat-modified lipoplexes is dependent on the amount of cationic lipid in the lipoplexes and on the way, Tat was coupled to the lipoplexes. Moreover, it was shown that the cellular uptake of both Tat-modified and un-modified lipoplexes was very fast and, in contrast to previous publications, temperature-dependent [Hyndman et al., 2004]. A concept called "Programmed Packaging" was proposed by Kogure et al., who developed a multi-functional envelope-type nano device (MEND), consisting of a condensed DNA core and a surrounding lipid envelope. This packaging method involves three steps: (1) DNA condensation with a polycation, (2) lipid film hydration for the electrostatic binding of the condensed DNA and (3) sonication to package the condensed DNA with lipids. MEND, having octa-arginine on the envelope for enhancing cellular uptake, showed a 1000-fold higher transfection activity than a DNA/poly-L-lysine/lipid complex prepared in similar conditions [Kogure et al., 2004]. Another study, by Khalil et al., also described the high-efficiency delivery of nucleic acids to eukaryotic cells using MEND particles containing polycation-condensed nucleic acids encapsulated in an R8-DOPE lipid envelope. MEND particles were shown to be non-cytotoxic and achieved transfection efficiencies as high as adenovirus [Khalil et al., 2010]. In this case, the high efficiency of MEND particles was ascribed, at least in part, to R8 which was claimed to promote cellular uptake by macropinocytosis, improving intracellular trafficking towards more efficient gene expression. Along the same lines, work of the same research group demonstrated that gene expression of condensed plasmid DNA encapsulated in R8-modified nanoparticles was more than one order of magnitude higher than that of K8modified nanoparticles, and two orders of magnitude higher than gene expression using unmodified nanoparticles. Differences in gene expression achieved with R8- and K8-modified liposomes could not be attributed to differences in cellular uptake, since both kinds of complexes were taken up primarily via macropinocytosis at comparable efficiencies. Moreover, it was described that modification of nanoparticles with a high density of R8 allows their escape from endocytotic vesicles via membrane fusion at both acidic and neutral $\mathrm{pH}$, and that the guanidinium groups of arginine residues, and not only their positive charge, are important for efficient endosomal escape [Trabulo et al., 2010]. Recently, MacKay et al. described gene transfer using PEGylated bio-responsive nano-lipid particles (NLPs) containing plasmid DNA. In this study, the Tat peptide was attached either directly to a phospholipid (Tatp-lipid) or via a $2 \mathrm{kDa}$ PEG (Tatp-PEG-lipid); incorporation of $0.3 \mathrm{~mol} \%$ Tatp-PEG into $\mathrm{pH}$-sensitive NLPs improved transfection 100,000-fold compared to NLPs. Although, Tatp-PEG-lipid could dramatically increase gene expression in vitro, when tested in brain and in implanted tumors, a restriction of NLP distribution to the vicinity of the infusion catheter reduced the absolute level of gene transfer [MacKay et al., 2008]. 
Over the last years, a research group focused on the S413-PV cell-penetrating peptide generated from the combination of 13-amino acid cell penetrating sequence derived from the Dermaseptin S4 peptide with the SV40 large T antigen nuclear localization signal [Trabulo et al., 2010]. In these studies, complexes obtained through electrostatic association of the S413PV cell penetrating peptide with plasmid DNA are able to very efficiently mediate transfection, particularly at high peptide/DNA charge ratios (5/1 and higher). Importantly, complexes prepared with the S413-PV or reverse NLS peptides mediate transfection at significantly higher efficiencies than those containing the scrambled version of the peptide, demonstrating the importance of the cell-penetrating sequence derived from the Dermaseptin S4 peptide (amino acids 1-13) to the transfection process. Additionally, we demonstrated that ternary complexes, resulting from association of cationic liposomes to peptide/DNA complexes, are significantly more efficient in mediating transfection than the corresponding peptide/DNA or cationic liposome/DNA complexes [Trabulo et al., 2010]. In agreement with what has been described for oligonucleotides, CPPs seem to be very efficient to mediate the uptake of plasmid DNA, as well as lipoplexes and polyplexes containing DNA, surpassing the cell membrane barrier. However, the challenge of overcoming the entrapment of complexes inside endosomes has not been solved as easily as initially predicted, even taking advantage of the capacity of direct translocation to the cytoplasm of some CPPs. Nevertheless, several of the studies described above present promising strategies to overcome this limitation, such as chemical modification of the peptide backbone or coupling of CPPs to other classes of delivery vectors. Overall, accumulated evidence suggests that CPPs used in combination with other delivery systems are more likely to be effective for gene therapy purposes than CPPs alone [Trabulo et al., 2010].

\section{Bioactive peptides}

As antibiotic resistance increases worldwide, there is an increasing pressure to develop novel classes of antimicrobial compounds to fight infectious disease. Peptide therapeutic represents a novel class of therapeutic agents. Some of them, such as cationic antimicrobial peptides (CAMPs) and peptidoglycan recognition proteins (PGRPs), have been identified from studies of innate immune effector mechanisms, while others are completely novel compounds generated in biological systems. Currently, only selected cationic antimicrobial peptides have been licensed, and for topical applications. However, research using new approaches to identify novel antimicrobial peptide therapeutics, and new approaches to delivery and improving stability, will result in an increased range of peptide therapeutics available in the clinic for broader applications. A potentially rich source of peptide therapeutics that is being investigated by researchers is the innate immune response, the effectors of which are produced by eukaryotes to defend themselves against microbial attack [Oyston et al., 2009].

Human cancer is one of the most important causes of death in the western countries. In advanced stages of the disease, the therapeutic opportunities are still limited due to the difficulty to target specifically only cancer cells sparing healthy ones. Cancer cells have on their surface antigens that are expressed at higher levels than their normal counterparts. Often these antigens (also called tumor-associated antigens) have receptor activity and bind to specific 
proteins or peptides. The latter can be used for the specific delivery of anticancer drugs to cancer cells through retargeting strategies and/or for the direct modulation of cancer cell proliferation and survival interacting with cell-surface-specific receptors. These bioactive peptides can be raised against either tumor cells themselves or to the tumor microenvironment cell components (tumor vessels, tumor-associated macrophages, and fibroblasts) [Fields et al., 2009]. However, the feasibility of pharmacological application of peptides depends on absorption and bioavailability in intact forms in target tissues.

Moreover, their correct bio-distribution is sometimes hindered by biopharmaceutical obstacles, that is, protection by circulating protease-mediated degradation or specific accumulation in tumor tissues. Chemical modification of peptide backbone can increase the stability of peptides in biological fluids. Moreover, the use of delivery systems, and in particular the use of nanotechnologies, not only protects peptides from enzymatic degradation but also improves the delivery of the bioactive peptide in the target tissue. Moreover, peptide conjugation on the surface of nano-vectors can be useful for selective delivery of conventional chemotherapeutic agents in tumor tissues. The requirements for an effective and safe dermatological therapeutic or active ingredient are included as following:

1. The molecule exhibits a proven specific beneficial bioactivity that would lead to a rational demonstrable effect.

2. The bioactivity does not have a negative consequence either theoretically or experimentally due to its mechanism of action.

3. The molecule does not exhibit toxicity such as cytotoxicity, inflammation, immunogenicity, or mutagenicity.

4. The molecule is capable of reaching its desired target intact and in its active form.

5. The molecule can be formulated in such a way as to be stable, compatible with other components, and be delivered effectively to the skin [Fields et al., 2009].

Collectively, these are not easily achieved criteria. For a new technology paradigm to emerge, these criteria not only have to be met but be applicable across the wide range of product-acceptable bioactivities. Peptides have significant advantages over many other technologies in addressing these criteria primarily based upon their chemistry. Peptides consist of chains of amino acids which can be modified in many ways to increase receptor binding, increase specificity, decrease toxicity, and increase skin penetration, stability, and solubility. In this way, the field of bioactive peptides for dermatological applications has changed significantly in recent years. From modest beginnings of a single peptide capable of stimulating collagen, technological advances have created newer peptides capable of targeting most aspects of dermal health. These advances include neutralizing toxins, stimulating fibroblast scaffolding, reducing inflammation and other desirable effects [Fields et al., 2009].

\section{Challenges in gene delivery for DNA vaccines}

The goal of DNA vaccination is transfection of an antigen presenting cells (APC) or a bystander cell to produce antigens in an immuno-stimulatory setting. The field of genetic vaccines has 
so far been limited by a lack of safe and effective gene delivery systems [Nguyen et al., 2008]. We attempted to mention some barriers and solutions (e.g., viral/non-viral methods) for DNA vaccines individually.

\subsection{Viral and non-viral methods for gene delivery}

The main recombinant viral vectors used for gene delivery are adenovirus, adeno-associated virus (AAVs), retrovirus and lentivirus. The advantages of adenovirus are infection of a wide range of human cell types, ability to infect non-dividing cells and lower risk of insertional mutagenesis. However, adenovirus expression is short lived and adenoviruses can cause a severe, even lethal, inflammatory response due to prior immune exposure. AAV, which depends on adenovirus or another virus for replication, has also been used for gene delivery with the advantages of predictable chromosomal insertion and no known pathological consequence of infection [Nguyen et al., 2008]. The main advantage of retroviruses, their ability to integrate into the host genome for long-term expression, is also their main disadvantage as this integration can cause mutagenesis and potentially cancer. Retroviruses are also further limited by their inability to infect non-dividing cells. Lentiviruses, which can transfect a broad spectrum of cell types, are the most efficient method to transfect DCs in vitro and in vivo. Yang et al. recently reported very high levels of immune activation and therapeutic tumor rejection following immunization with a lentiviral vector engineered to target DCs by the cell surface receptor DC-SIGN [Yang et al., 2008]. In particular, Merck \& Co. has advanced the use of viral DNA vaccines for HIV vaccination. While there have been some successes in using viral gene therapy and many clinical trials are currently ongoing there are currently no approved protocols. Problems with viral delivery systems include immunologic priming to the vector itself, oncogenicity due to insertional mutagenesis, difficult manufacturing and limited DNA cargo capacity [Nguyen et al., 2008]. Clinical trials have highlighted some of these safety risks as viral gene delivery has resulted in both cancer and deaths. Recently, Merck \& Co. stopped its Phase III HIV adenovirus vaccine prompting renewed questions about the utility of viral vectors. The safety challenges and limitations of viral vectors have resulted in increased interest in non-viral approaches to gene delivery using non-viral materials. In general, the nonviral methods of DNA vaccination utilized in clinical trials, recently reviewed by Lu et al., rely on physical methods. Injection of naked DNA plasmids has found limited success in humans particularly when injected intramuscularly, even though in smaller animal models naked DNA vaccination produces robust humoral and cell-mediated responses [ $\mathrm{Lu}$ et al., 2008]. However, the rapid degradation/clearance [half-life of under $5 \mathrm{~min}$ if injected intravenously (IV)] of unprotected nucleic acids, poor induction of humoral immune responses in DNA vaccination in larger animals and requirement for large doses has hindered progress into clinical trials. Clinically, relevant physical methods that have been employed include electroporation, ballistics (gene gun), ultrasound and magnetofection. Encapsulation or complexation of DNA with a biomaterial can significantly enhance DNA stability, cellular uptake of DNA and final protein expression. Materials shown to possess potential for the delivery of genes include inorganic nanoparticles and surfaces that bind to or encapsulate DNA [Lu et al., 2008; Nguyen et al., 2008]. Cationic biomolecules including lipids, polysaccharides, polymers, and dendrimers can also electrostatically complex anionic DNA to facilitate transfection. Unless specifically 
designed to do so, DNA delivered non-virally has low potential for genomic integration [Nguyen et al., 2008]. Non-viral delivery systems for gene therapy are generally cheaper to manufacture, easily scalable from laboratory to GMP-scale production and are typically more robust for long-term storage compared to their viral counterparts. Despite achieving greater efficacy than naked DNA administration (IM, IV or otherwise), physical methods for gene delivery are often limited due to local tissue damage and insufficient gene expression. Research into non-viral gene delivery has been ongoing since the 1970s, and as understanding of the mechanisms of gene delivery has grown, the design of synthetic biomaterials has become more advanced. However, while there have been advances, non-viral methods of gene delivery generally still have lower efficacy than viruses [Nguyen et al., 2008].

\subsection{Barriers to gene delivery for DNA vaccines}

There are many potential blocks that must be overcome for successful DNA delivery, a process broadly defined as transfection. Plasmid DNA must first be packaged into particles. Requirements for gene delivery include protection of plasmid DNA from degradation, localization to the tissue and cell types of interest avoiding off-target distribution, minimal inactivation by interaction with serum proteins, low clearance from the blood or interstitial space and efficient transport through the extracellular matrix to the surface of target cells. Next, the DNA-containing particles must associate with cells and become internalized into them by cellular uptake processes [Nguyen et al., 2008]. Following uptake, DNA-containing particles must escape the endosomal/ phagosomal compartment into the cytoplasm and release their DNA cargo. DNA must finally translocate into the nucleus to be transcribed into mRNA and subsequently translated into protein antigen. Viruses have evolved to accomplish these steps and provide a framework for the design of synthetic delivery particles. Despite efficient uptake of particles of a variety of sizes, in vitro and in vivo transfection of DCs is still notoriously difficult to achieve. APCs are specialized not only for uptake of antigen but also rapid and efficient antigen processing. As a key role of APCs is to internalize and process pathogens for immune activation, APCs may have greater protection against foreign (viral) DNA entry into the nucleus, which may be a barrier to DNA vaccination. Whereas in vitro investigation of transfection efficiency in cell culture can be used to identify promising materials for transfection, there exist multiple extracellular barriers to effective DNA vaccination in vivo. DCs reside in the blood, in the skin (Langerhans cells), other mucosal barriers, and in lymph nodes [Nguyen et al., 2008]. macrophages (MPs) also exist in lymph nodes, as circulating precursors in the blood (monocytes) that differentiate as they enter inflamed tissue cites, and as specialized MPs lining the spleen and liver (Kupfer cells) forming the phagocytic part of the reticuloendothelial system (RES). Access to these APCs is therefore determined not only by route of injection, but also by ability of a particle to drain into lymphatic systems or activate inflammatory signals to recruit APCs. For example, Reddy et al. have illustrated size-based targeting of lymph-node resident DCs by accessing lymphatic vessels with $25 \mathrm{~nm}$ particles; lymphatic drainage and DC uptake was significantly reduced with $100 \mathrm{~nm}$ particles [Reddy et al., 2007]. Many cationic delivery materials, both polymeric and lipid, form vector-nucleic acid particle (VNP) complexes by electrostatic interactions with the negative charges on the phosphate groups of the DNA backbone. A net positive surface charge can facilitate transfection by interacting with the negatively charged glycoproteins at the cell mem- 
brane. However, electrostatic interactions can also rapidly lead to aggregation of these VNPs with serum proteins; as VNP-protein aggregate size increases they can be eliminated from circulation by the phagocytic MPs of the RES, deposit non-specifically in microvascular beds or crash out of solution, which may cause acute toxicity [Nguyen et al., 2008]. In addition to containing high concentrations of negatively charged proteins, plasma also has a significant ionic strength. Interactions between serum proteins, blood and interstitial fluid solutes and polycationic carrier materials can lead to competitive binding, destabilization of the VNP and subsequent premature release of the nucleic acid payload. Interaction with complement proteins, C3 and $\mathrm{C} 4$ in particular, can activate the innate immune responses resulting in acute inflammation and lead to severe acute toxicity or death. Mucosal surfaces and serum also contain DNase and RNase enzymes that specifically degrade nucleic acids. Condensed VNPs prevent the degradation of the nucleic acid payload by steric inhibition of these DNases/RNases. The addition of PEG and other hydrophilic polymers can be used to prevent aggregation with serum proteins and subsequent rapid clearance [Nguyen et al., 2008]. This simple functionality can sharply increase the serum half-life of a particle and prevent acute toxic events due to non-specific interactions, but also results in lower transfection efficiency and reduced cellular targeting. Toxicity at the cellular level and/or due to interactions with the immune system, liver, kidneys, or other complex organ systems can be a concern with non-viral gene delivery. For example, PEI has been shown to be an effective transfection agent but has also been reported to be toxic in animal models. Polycations such as PEI and cationic lipids such as 1, 2-dioleoyl-3-trimethylammonium-propane (DOTAP) also tend to activate complement and the reticuloendothelial system (RES) aggregate with serum proteins, and can aggregate with red blood cells as well. Some toxicity issues both in vitro and in vivo can be addressed by chemical modification of PEI [Nguyen et al., 2008]. For increased safety, biodegradable gene delivery systems have also been developed, including degradable cross-linked PEI [Kim et al., 2005], poly (ortho-esters) (POE) [Heller et al., 2000] and poly (b-amino esters) (PBAE) [Anderson et al., 2003].

\subsection{Particle uptake by APCs and targeting gene delivery for DNA vaccines}

Particle uptake by phagocytosis (particles $>500 \mathrm{~nm}$ ), macropinocytosis, and receptor-mediated endocytosis are particularly important routes of entry into APCs. Delivery systems can be designed to exploit these ways. Cell-specific targeting can significantly enhance transfection efficiency and the desired therapeutic outcome. The direct conjugation of targeting moieties such as receptor ligands, peptides, sugars, aptamers and antibodies can increase cell and tissue specificity and transfection efficiency. Additionally, targeting can be based upon size-specific signals to avoid off-target affects. A variety of strategies exist for targeting APCs. First, APCs express Fc-receptors, which bind to the constant region of antibodies to facilitate uptake of antibody-coated foreign bodies. APCs also express complement receptors that help clear complement-opsonized particles [Nguyen et al., 2008]. Lectin-binding receptors, such as the mannose receptor and scavenger receptors that recognize apoptotic bodies, certain bacterial components and other non-self motifs are PRRs commonly found on APCs that can enhance particle uptake and may trigger innate immune activation. Second, unlike most other cell types, immature DCs constitutively sample their extracellular fluid environment nonspecifically through macropinocytosis to maintain immune surveillance and vigilance for 
foreign particles, as well as to present endogenous proteins for maintenance of self-tolerance. This constant sampling may explain DC-targeting by particle size. Particle size may also influence the specific route of entry, as reviewed recently by Xiang et al. Particle surface characteristics also play a role in uptake, as cationic particles more readily associate with the negatively charged glycoproteins on the cell surface and promote non-specific uptake, and other surface characteristics can activate opsonization [Xiang et al., 2006]. Champion et al. have clarified the role of particle shape in influencing phagocytosis by MPs, as well as provided simple methods for creating materials with complex shapes and sizes to take advantage of particle physical properties. While spherical micro- and nano-particles are efficiently phagocytosed by lung alveolar MPs, phagocytosis can be inhibited by contact with odd geometries due to an inability to form the necessary actin structures. In general, size plays a more significant role with particle association with the cell surface than with internalization. These studies suggest a role for particle surface nano and micro-structure in the design of APCtargeted DNA vaccine delivery systems [Champion et al., 2007].

\subsection{Biomaterials for DNA delivery: Non-polymeric biomaterials for gene delivery}

Many materials have been developed for gene delivery. Early chemical methods of increasing the efficacy of gene delivery focused on co-precipitation of the DNA with salts such as calcium phosphate. More recently, inorganic materials have also been combined with polymers to form hybrid gene delivery nanoparticles. For example, textured surfaces and silica nano-particles have been shown to be effective for gene transfer in vitro and organically modified silica nanoparticles have been shown to deliver genes in vivo. Gold nano-particles have also been combined with PEI for hybrid gene delivery systems. Cationic lipids have been the non-viral gene delivery vectors of choice for clinical application since Felgner first introduced their use in 1987. The cationic lipid molecule consists of a hydrophilic positively charged head group, a linker that may impart some functionality such as $\mathrm{pH}$ sensitivity and a hydrophobic long chain tail [Felgner et al., 1987]. A prototypical cationic lipid is DOTAP; it is the most widely used lipid for gene delivery. For in vivo delivery, nucleic acids are usually encapsulated into liposomes: vesicles with lipid bilayer membranes that exist as large uni-lamellar vesicles (LUVs) or multi-lamellar vesicles (MLVs). Liposomes generally consist of a single cationic lipid or a mixture of cationic lipids that facilitate nucleic acid binding and transfection, cholesterol or diolelphophatidylethanolamine (DOPE) to impart some rigidity or stability to the complex, and PEG to shield particles from aggregation, serum components, or other non-specific interactions. Lipids have been used extensively in gene therapy and are the main non-viral delivery vectors used in clinical trials. Unfortunately, some lipoplexes are toxic, interact nonspecifically with serum proteins and cells, aggregate quickly, activate the complement system, or have low in vivo efficacy. One promising approach that may address these problems is to increase the chemical diversity of lipid-like materials through combinatorial synthesis approaches [Nguyen et al., 2008].

\section{Conclusion}

Gene therapy possesses great potential for combating a variety of diseases. Initial results are promising and some technologies have advanced to clinical trials. Yet challenges remain, and 
despite decades of study, safe and efficient gene delivery remains a major blockage in human medicine. The current progresses of gene therapy are further focused on synthesized nanoparticle technologies such as polymers (PLGA, chitosan and PEI), lipids and peptides. It is necessary to understand the effects of particle size, surface characteristics and material interactions. Research on cell penetrating peptides (CPPs) as gene/ drug delivery systems has clarified their capacity to promote the efficient internalization of therapeutic biomolecules. Despite differences in size, charge and/or structure between different bioactive molecules, it is clear that CPP-based systems appear to be very versatile and efficient delivery is achievable following proper adjustment of the carrier to the transported biomolecule. Because the development of drug, oligonucleotide or gene delivery systems is aimed at a clinical application, the design of these novel delivery vectors should consider other important issues including safety, bio-distribution, ease of manufacturing, scale-up, reproducibility and analytical and physical characterization. The advance of CPP technology depends on the development of strategies that facilitate endosomal escape and that confer cell specificity to these systems. A careful investigation of the mechanisms of internalization of CPP-cargo complexes or conjugates will greatly help the improvement of this powerful technology. However, a viable non-viral gene vector for systemic delivery depends on its capacity to bypass a series of physiological barriers and its efficiency in carrying nucleic acids to a targeted site within a cell. The concept of a multifunctional delivery system helps to solve the problems associated with various barriers. The availability of delivery devices directed towards each individual barrier, provides a basis for this direction, although the complexity in the development of multifunctional non-viral vectors is much more than a merely combination of various devices into a single system. In order to achieve the optimal gene delivery, the researchers have focused largely on the evolution of "intelligent" bio-responsive materials, as well as on the advances in formulation technologies. In this process, a number of strategies have emerged including the balances between gene packing and controlled release, and optimal control between long-circulation and intracellular trafficking that promotes safer and more efficient delivery of gene/drug in a systemic context.

\section{Author details}

Azam Bolhassani ${ }^{1}$ and Tayebeh Saleh ${ }^{2}$

*Address all correspondence to: azam_bolhassani@yahoo.com

*Address all correspondence to: A_bolhasani@pasteur.ac.ir

1 Molecular Immunology and Vaccine Research Lab., Pasteur Institute of Iran, Tehran, Iran

2 Department of Nanobiotechnology, Faculty of Biological Sciences, Tarbiat Modares University, Tehran, Iran 


\section{References}

[1] Abbott, A. (1992). Gene therapy: Italians first to use stem cells. Nature, , 356(6369), 465-499.

[2] Alexis, F, \& Basto, P. Levy-Nissenbaum, E.; Radovic-Moreno, A.F.; Zhang, L.; Pridgen, E.; Wang, A.Z.; Marein, S.L.; Westerhof, K. \& Molnar, L.K. ((2008). HER-2-targeted nanoparticle-affibody bioconjugates for cancer therapy. Chem.Med.Chem., , 3, 1839-1843.

[3] Anderson, D. G, Lynn, D. M, \& Langer, R. (2003). Semi-automated synthesis and screening of a large library of degradable cationic polymers for gene delivery. Angew. Chem. Int. Ed. Engl., , 42(27), 3153-3158.

[4] Askoxylakis, V, Zitzmann, S, Mier, W, Graham, K, Krämer, S, Von Wegner, F, Fink, R. H. A, Schwab, M, Eisenhut, M, \& Haberkorn, U. (2005). Preclinical evaluation of the breast cancer cell-binding peptide, p160. Clinical cancer research, , 11, 6705-6712.

[5] Bauhuber, S, Hozsa, C, Breunig, M, \& Göpferich, A. (2009). Delivery of nucleic acids via disulfide-based carrier systems. Advanced Materials, , 21, 3286-3306.

[6] Benoit, D. S. W, Henry, S. M, Shubin, A. D, Hoffman, A. S, \& Stayton, P. S. (2010). pHresponsive polymeric sirna carriers sensitize multidrug resistant ovarian cancer cells to doxorubicin via knockdown of polo-like kinase. Molecular pharmaceutics, , 7, 442-455.

[7] Bhaskar, S, Tian, F, Stoeger, T, Kreyling, W, Fuente, J. M, Grazú, V, Borm, P, Estrada, G, Ntziachristos, G, \& Razansky, D. (2010). Multifunctional nanocarriers for diagnostics, drug delivery and targeted treatment across blood-brain barrier: perspectives on tracking and neuroimaging. Particle and Fibre Toxicology, , 7, 3.

[8] Blaese, R. M, Culver, K. W, Miller, A. D, Carter, C. S, Fleisher, T, Clerici, M, Shearer, G, Chang, L, Chiang, Y, Tolstoshev, P, Greenblatt, J. J, Rosenberg, S. A, Klein, H, Berger, M, Mullen, C. A, Ramsey, W. J, Muul, L, Morgan, R. A, \& Anderson, W. F. gene therapy for ADA- SCID: initial trial results after 4 years. Science, , 270(5235), 475-480.

[9] Bolhassani, A. (2011). Potential efficacy of cell-penetrating peptides for nucleic acid and drug delivery in cancer. BBA-Reviews on Cancer, , 1816, 232-246.

[10] Bolhassani, A, Safaiyan, S, \& Rafati, S. (2011). Improvement of different vaccine delivery systems for cancer therapy. Molecular Cancer, , 10(3), 1-20.

[11] Branden, L. J, Mohamed, A. J, \& Smith, C. I. (1999). A peptide nucleic acid-nuclear localization signal fusion that mediates nuclear transport of DNA. Nat. Biotechnol., , $17,784-787$.

[12] Cavazzana-calvo, M, Thrasher, A, \& Mavilio, F. (2004). The future of gene therapy. Nature, , 427(6977), 779-781.

[13] Cell therapy fights leukaemia. (2011). http://www.nature.com/news. 
[14] Chai, C, Xie, Z, \& Grotewold, E. (2011). SELEX (Systematic Evolution of Ligands by EXponential Enrichment) as a powerful tool for deciphering the protein-DNA interaction space. Methods Mol. Biol., , 754, 249-258.

[15] Champion, J. A, Katare, Y. K, \& Mitragotri, S. (2007). Particle shape: a new design parameter for micro- and nanoscale drug delivery carriers. J. Control. Release, , 121(1-2), 3-9.

[16] Cheng, R, Feng, F, Meng, F, Deng, C, Feijen, J, \& Zhong, Z. (2011). Glutathioneresponsive nano-vehicles as a promising platform for targeted intracellular drug and gene delivery. Journal of Controlled Release. , 152(1), 2-12.

[17] De Coupade, C, Fittipaldi, A, Chagnas, V, Michel, M, Carlier, S, Tasciotti, E, Darmon, A, Ravel, D, Kearsey, J, Giacca, M, \& Cailler, F. (2005). Novel human-derived cellpenetrating peptides for specific subcellular delivery of therapeutic biomolecules. Biochem. J., , 390, 407-418.

[18] DNA nanoballs boost gene therapy(2002). New Scientist, http://www.newscientist.com/article/dndna-nanoballs-boost-gene-therapy.html.

[19] Du, F. S, Wang, Y, Zhang, R, \& Li, Z. C. (2010). Intelligent nucleic acid delivery systems based on stimuli-responsive polymers. Soft Matter, , 6, 835-848.

[20] El-Andaloussi, S, Johansson, H. J, Holm, T, \& Langel, U. (2007). A novel cell-penetrating peptide, M918, for efficient delivery of proteins and peptide nucleic acids. Mol. Ther. , $15,1820-1826$.

[21] Eliyahu, H, Barenholz, Y, \& Domb, A. J. (2005). Polymers for DNA delivery. Molecules, , $10,34-64$.

[22] Farokhzad, O. C, Jon, S, Khademhosseini, A, Tran, T. N. T, La, D. A, \& Langer, R. (2004). Nanoparticle-aptamer bioconjugates. Cancer research, , 64, 7668.

[23] Farokhzad, O. C, Cheng, J, Teply, B. A, Sherifi, I, Jon, S, Kantoff, P. W, Richie, J. P, \& Langer, R. (2006). Targeted nanoparticle-aptamer bioconjugates for cancer chemotherapy in vivo. Proceedings of the National Academy of Sciences, , 103, 6315-6320.

[24] Fawell, S, Seery, J, Daikh, Y, Moore, C, Chen, L. L, Pepinsky, B, \& Barsoum, J. (1994). Tat-mediated delivery of heterologous proteins into cells. Proc. Natl. Acad. Sci. U.S.A., , 91, 664-668.

[25] Felgner, P. L, Gadek, T. R, Holm, M, Roman, R, Chan, H. W, Wenz, M, Northrop, J. P, Ringold, G. M, \& Danielsen, M. (1987). Lipofection: a highly efficient, lipid-mediated DNA-transfection procedure. Proc. Natl. Acad. Sci. USA, , 84(21), 7413-7417.

[26] Fields, K, Falla, T. J, Rodan, K, \& Bush, L. (2009). Bioactive peptides: signaling the future. Journal of Cosmetic Dermatology, , 8, 8-13.

[27] Fisher, J. (1995). Murine gene therapy corrects symptoms of sickle cell disease". Magazine of the Life Sciences. 
[28] Fittipaldi, A, Ferrari, A, Zoppe, M, Arcangeli, C, Pellegrini, V, Beltram, F, \& Giacca, M. (2003). Cell membrane lipid rafts mediate caveolar endocytosis of HIV-1 Tat fusion proteins. J. Biol. Chem., , 278, 34141-34149.

[29] Frankel, A. D, \& Pabo, C. O. (1998). Cellular uptake of the tat protein from human immunodeficiency virus. Cell, , 55, 1189-1193.

[30] Friedmann, T, \& Roblin, R. (1972). Gene therapy for human genetic disease. Science, , 175(4025), 949.

[31] Ganta, S, Devalapally, H, Shahiwala, A, \& Amiji, M. (2008). A review of stimuliresponsive nanocarriers for drug and gene delivery. Journal of Controlled Release, , $126,187-204$.

[32] GaoX; Kim, K.S. \& Liu, D. ((2007). Non-viral gene delivery: What we know and what is next. A.A.P.S. Journal, , 9(1), E92-E104.

[33] Gene therapy may switch off Huntington's(2003). New Scientist, http://www.newscientist.com

[34] Gene therapy first for poor sight(2007). BBC News, http://news.bbc.co.uk

[35] Green, M, \& Loewenstein, P. M. (1988). Autonomous functional domains of chemically synthesized human immunodeficiency virus Tat trans-activator protein. Cell, , 55, 1179-1188.

[36] Gu, F. X, Karnik, R, Wang, A. Z, Alexis, F, Levy-nissenbaum, E, Hong, S, Langer, R. S, \& Farokhzad, O. C. (2007). Targeted nanoparticles for cancer therapy. Nano Today, , 2, 14-21.

[37] Hahn, W, Pyun, W. B, Kim, D. S, Yoo, W. S, Lee, S. D, Won, J. H, Shin, G. J, Kim, J. M, \& Kim, S. (2011). Enhanced cardioprotective effects by co-expression of two isoforms of hepatocyte growth factor from naked plasmid DNA in a rat ischemic heart disease model". The Journal of Gene Medicine, , 13(10), 549-555.

[38] Heller, J, Barr, J, Ng, S. Y, Shen, H. R, Schwach-abdellaoui, K, Einmahl, S, Rothenweinhold, A, \& Gurny, R. (2000). Poly(ortho esters)- their development and some recent applications. Eur. J. Pharm. Biopharm., , 50(1), 121-128.

[39] Hyndman, L, Lemoine, J. L, Huang, L, Porteous, D. J, Boyd, A. C, \& Nan, X. (2004). HIV-1 Tat protein transduction domain peptide facilitates gene transfer in combination with cationic liposomes. J. Control. Release, , 99, 435-444.

[40] Ignatovich, I. A, Dizhe, E. B, Pavlotskaya, A. V, Akifiev, B. N, Burov, S. V, Orlov, S. V, \& Perevozchikov, A. P. (2003). Complexes of plasmid DNA with basic domain 47-57 of the HIV-1 Tat protein are transferred to mammalian cells by endocytosis-mediated pathways. J. Biol. Chem., , 278, 42625-42636.

[41] Imai, K, \& Takaoka, A. (2006). Comparing antibody and small-molecule therapies for cancer. Nature Reviews-Cancer, , 6, 714-727. 
[42] Jabr-milane, L, Van Vlerken, L, Devalapally, H, Shenoy, D, Komareddy, S, Bhavsar, M, \& Amiji, M. (2008). Multi-functional nanocarriers for targeted delivery of drugs and genes. Journal of Controlled Release, , 130, 121-128.

[43] Joliot, A. H, Triller, A, Volovitch, M, Pernelle, C, \& Prochiantz, A. (1991). alpha-2, 8polysialic acid is the neuronal surface receptor of antennapedia homeobox peptide. New Biol., , 3, 1121-1134.

[44] Kaiser, J. (2009). Gene therapy halts brain disease in two boys", Science

[45] Kam, N. W. S, Liu, Z, \& Dai, H. (2005). Functionalization of carbon nanotubes via cleavable disulfide bonds for efficient intracellular delivery of siRNA and potent gene silencing. Journal of the American Chemical Society, , 127, 12492-12493.

[46] Kang, H. C, Kang, H. J, \& Bae, Y. H. (2011). A reducible polycationic gene vector derived from thiolated low molecular weight branched polyethyleneimine linked by 2iminothiolane. Biomaterials, , 32, 1193-1203.

[47] Khalil, I. A, Futaki, S, Niwa, M, Baba, Y, Kaji, N, Kamiya, H, \& Harashima, H. (2004). Mechanism of improved gene transfer by the N-terminal stearylation of octaarginine: enhanced cellular association by hydrophobic core formation. Gene Ther., , 11, 636-644.

[48] Kilk, K, Andaloussi, S, Jarver, P, Meikas, A, Valkna, A, Bartfai, T, Kogerman, P, Metsis, M, \& Langel, U. (2005). Evaluation of transportan 10 in PEI mediated plasmid delivery assay. J. Control. Release, , 103, 511-523.

[49] Kim, Y. H, Park, J. H, Lee, M, Kim, Y. H, Park, T. G, \& Kim, S. W. (2005). Polyethylenimine with acid-labile linkages as a biodegradable gene carrier. J. Control. Release, , 103(1), 209-219.

[50] Kim, S. H, Mok, H, Jeong, J. H, Kim, S. W, \& Park, T. G. (2006). Comparative evaluation of target-specific GFP gene silencing efficiencies for antisense ODN, synthetic siRNA, and siRNA plasmid complexed with PEI-PEG-FOL conjugate. Bioconjugate chemistry, , 17, 241-244.

[51] Kim, Y. H, Park, J. H, Lee, M, Park, T. G, \& Kim, S. W. (2005). Polyethylenimine with acid-labile linkages as a biodegradable gene carrier. Journal of Controlled Release, , 103, 209-219.

[52] Kim, T, \& Kim, S. W. (2011). Bio-reducible polymers for gene delivery. Reactive and functional polymers, , 71, 344-349.

[53] Kleemann, E, Neu, M, Jekel, N, Fink, L, Schmehl, T, Gessler, T, Seeger, W, \& Kissel, T. (2005). Nano-carriers for DNA delivery to the lung based upon a TAT-derived peptide covalently coupled to PEG-PEI. J. Control. Release, , 109, 299-316.

[54] Knorr, V, Allmendinger, L, Walker, G. F, Paintner, F. F, \& Wagner, E. (2007). An acetalbased PEGylation reagent for $\mathrm{pH}$-sensitive shielding of DNA polyplexes. Bioconjugate chemistry, , 18, 1218-1225. 
[55] Kogure, K, Moriguchi, R, Sasaki, K, Ueno, M, Futaki, S, \& Harashima, H. (2004). Development of a non-viral multifunctional envelope-type nano device by a novel lipid film hydration method. J. Control. Release, , 98, 317-323.

[56] Komáromy, A, Alexander, J, Rowlan, J, Garcia, M, Chiodo, V, Kaya, A, Tanaka, J, Acland, G. M, Hauswirth, W. W, \& Aguirre, G. D. (2010). Gene therapy rescues cone function in congenital achromatopsia. Human Molecular Genetics, , 19(13), 2581-2593.

[57] Korthof, G. (1999). The implications of Steele's soma-to-germline feedback for human gene therapy, http://home.planet.nl

[58] Langel, U. (2002). Cell-penetrating peptides: Processes and Applications. CRC Press, Amazon.com Textbooks

[59] Lee, H, Mok, H, Lee, S, Oh, Y. K, \& Park, T. G. (2007). Target-specific intracellular delivery of siRNA using degradable hyaluronic acid nanogels. Journal of Controlled Release, , 119, 245-252.

[60] Lehner, R, Wang, X, Wolf, M, \& Hunziker, P. (2012). Designing switchable nanosystems for medical application. J. Control Release, , 161(2), 307-316.

[61] Levine, B. L, Humeau, L. M, \& Boyer, J. MacGregor, R.R.; Rebello T.; Lu, X.; Binder, G.K.; Slepushkin, V.; Lemiale, F.; Mascola, J.R.; Bushman, F.D.; Dropulic, B.; \& June, C.H. ((2006). Gene transfer in humans using a conditionally replicating lentiviral vector. Proc Natl Acad Sci U S A., , 103(46), 17372-17377.

[62] Lin, Y. L, Jiang, G, Birrell, L. K, \& Sayed, M. E. H. (2010). Degradable, pH-sensitive, membrane-destabilizing, comb-like polymers for intracellular delivery of nucleic acids. Biomaterials, , 31, 7150-7166.

[63] Lo, S. L, \& Wang, S. (2008). An endosomolytic Tat peptide produced by incorporation of histidine and cysteine residues as a nonviral vector for DNA transfection. Biomaterials, , 29, 2408-2414.

[64] Lomas, H, \& Canton, I. MacNeil, S.; Du, J.; Armes, S.P.; Ryan, A.J.; Lewis, A.L. \& Battaglia, G. ((2007). Biomimetic $\mathrm{pH}$ sensitive polymersomes for efficient DNA encapsulation and delivery. Advanced Materials, , 19, 4238-4243.

[65] Lu, S, Wang, S, \& Grimes-serrano, J. M. (2008). Current progress of DNA vaccine studies in humans. Expert Rev. Vaccines, , 7(2), 175-191.

[66] Ma, B, Zhang, S, Jiang, H, Zhao, B, \& Lv, H. (2007). Lipoplex morphologies and their influences on transfection efficiency in gene delivery. Journal of Controlled Release, , 123, 184-194.

[67] MacKayJ.A.; Li, W.; Huang, Z.; Dy, E.E.; Huynh, G.; Tihan, T.; Collins, R.; Deen, D.F. \& Szoka, F.C. ((2008). HIV TAT peptide modifies the distribution of DNA nanolipoparticles following convection-enhanced delivery. Mol. Ther., , 16, 893-900. 
[68] Maiolo, J. R, Ferrer, M, \& Ottinger, E. A. (2005). Effects of cargo molecules on the cellular uptake of arginine-rich cell-penetrating peptides. Biochim. Biophys. Acta, , 1712, 161-172.

[69] Manickam, D.S, \& Oupický, D. (2006). Multiblock reducible copolypeptides containing histidine-rich and nuclear localization sequences for gene delivery. Bioconjugate chemistry, , 17, 1395-1403.

[70] Manjappa, A. S, Chaudhari, K. R, Venkataraju, M. P, Dantuluri, P, Nanda, B, Sidda, C, \& Sawant, K. K. Ramachandra Murthy, R.S. ((2011). Antibody derivatization and conjugation strategies: application in preparation of stealth immunoliposome to target chemotherapeutics to tumor. Journal of Controlled Release, , 150, 2-22.

[71] Martin, M. E, \& Rice, K. G. (2007). Peptide-guided gene delivery. The AAPS journal, , 9, 18-29.

[72] Mastrobattista, E, Van Der Aa, M. A. E. M, Hennink, W. E, \& Crommelin, D. J. A. (2006). Artificial viruses: a nanotechnological approach to gene delivery. Nature Reviews Drug Discovery, , 5, 115-121.

[73] Mccormick, F. (2001). Cancer gene therapy: Fringe or cutting edge? Nature, , 1, 130-141.

[74] Mckenzie, D. L, Kwok, K. Y, \& Rice, K. G. (2000). A potent new class of reductively activated peptide gene delivery agents. Journal of Biological Chemistry, , 275, 9970-9977.

[75] Meng, F, Hennink, W. E, \& Zhong, Z. (2009). Reduction-sensitive polymers and bioconjugates for biomedical applications. Biomaterials, , 30, 2180-2198.

[76] Midoux, P, Pichon, C, Yaouanc, J. J, \& Jaffrès, P. A. (2009). Chemical vectors for gene delivery: a current review on polymers, peptides and lipids containing histidine or imidazole as nucleic acids carriers. Br. J. Pharmacol., , 157(2), 166-178.

[77] Morris, M. C, Chaloin, L, Mery, J, Heitz, F, \& Divita, G. (1999). A novel potent strategy for gene delivery using a single peptide vector as a carrier. Nucleic Acids Res.,, 27, 3510-3517.

[78] Murthy, N, Campbell, J, Fausto, N, Hoffman, A. S, \& Stayton, P. S. (2003). Design and synthesis of $\mathrm{pH}$-responsive polymeric carriers that target uptake and enhance the intracellular delivery of oligonucleotides. Journal of Controlled Release, , 89, 365-374.

[79] Nakase, I, Kobayashi, S, \& Futaki, S. (2010). Endosome-disruptive peptides for improving cytosolic delivery of bioactive macromolecules. Biopolymers, , 94, 763-770.

[80] Nguyen, D. N, Green, J. J, Chan, J. M, Langer, R, \& Anderson, D. G. (2008). Polymeric materials for gene delivery and DNA vaccination. Adv. Mater., , 20, 1-21.

[81] Nie, Y, Günther, M, Gu, Z, \& Wagner, E. (2011). Pyridylhydrazone-based PEGylation for $\mathrm{pH}$-reversible lipopolyplex shielding. Biomaterials, , 32, 858-869.

[82] Oishi, M, Nagasaki, Y, Itaka, K, Nishiyama, N, \& Kataoka, K. (2005). Lactosylated poly (ethylene glycol)-siRNA conjugate through acid-labile $\beta$-thiopropionate linkage to 
construct $\mathrm{pH}$-sensitive polyion complex micelles achieving enhanced gene silencing in hepatoma cells. Journal of the American Chemical Society, , 127, 1624-1625.

[83] Ogris, M, Walker, G, Blessing, T, Kircheis, R, Wolschek, M, \& Wagner, E. (2003). Tumortargeted gene therapy: strategies for the preparation of ligand-polyethylene glycolpolyethylenimine/ DNA complexes. Journal of Controlled Release, , 91, 173-181.

[84] Oyston, P. C. F, Fox, M. A, Richards, S. J, \& Clark, G. C. (2009). Novel peptide therapeutics for treatment of infections. Journal of Medical Microbiology, , 58, 977-987.

[85] Park, I. K, Singha, K, Arote, R. B, Choi, Y. J, Kim, W. J, \& Cho, C. S. (2010). pH-Responsive Polymers as Gene Carriers. Macromolecular rapid communications, , 31, 1122-1133.

[86] Peer, D, Karp, J. M, Hong, S, Farokhzad, O. C, Margalit, R, \& Langer, R. (2007). Nanocarriers as an emerging platform for cancer therapy. Nature nanotechnology, , 2, 751-760.

[87] Peng, Q, Zhong, Z, \& Zhuo, R. (2008). Disulfide cross-linked polyethylenimines (PEI) prepared via thiolation of low molecular weight PEI as highly efficient gene vectors. Bioconjugate chemistry, , 19, 499-506.

[88] Penn Medicine presents HIV gene therapy trial(2009). CROI, http://www.eurekalert.org

[89] Qiao, W, Wang, B, Wang, Y, Yang, L, Zhang, Y, \& Shao, P. (2010). Cancer therapy based on nanomaterials and nanocarrier systems. Journal of Nanomaterials, , 2010, 1-9.

[90] Read, M. L, Bremner, K. H, Oupický, D, Green, N. K, Searle, P. F, \& Seymour, L. W. (2003). Vectors based on reducible polycations facilitate intracellular release of nucleic acids. The journal of gene medicine, , 5, 232-245.

[91] Reddy, S. T, Van Der Vlies, A. J, Simeoni, E, Angeli, V, Randolph, G. J, Neil, O, Lee, C. P, Swartz, L. K, \& Hubbell, M. A. J.A. ((2007). Exploiting lymphatic transport and complement activation in nanoparticle vaccines. Nat. Biotechnol., , 25(10), 1159-1164.

[92] Richard, J. P, Melikov, K, Vives, E, Ramos, C, Verbeure, B, Gait, M. J, Chernomordik, L. V, \& Lebleu, B. (2003). Cell-penetrating peptides: A re-evaluation of the mechanism of cellular uptake. J. Biol. Chem. , 278, 585-590.

[93] Richard, J. P, Melikov, K, Brooks, H, Prevot, P, Lebleu, B, \& Chernomordik, L. V. (2005). Cellular uptake of un-conjugated Tat peptide involves clathrin-dependent endocytosis and heparin sulfate receptors. J. Biol. Chem. , 280, 15300-15306.

[94] Rittner, K, Benavente, A, Bompard-sorlet, A, Heitz, F, Divita, G, Brasseur, R, \& Jacobs, E. (2002). New basic membrane-destabilizing peptides for plasmid-based gene delivery in vitro and in vivo. Mol. Ther., , 5, 104-114.

[95] Roh, Y. H, Lee, J. B, Kiatwuthinon, P, Hartman, M. R, Cha, J. J, Um, S. H, Muller, D. A, \& Luo, D. (2011). DNAsomes: Multifunctional DNA-Based Nanocarriers. Small, , 7(1), 74-78. 
[96] Rogers, S. (1970). Gene therapy, New Sci., , 194.

[97] Rudolph, C, Plank, C, Lausier, J, Schillinger, U, Muller, R. H, \& Rosenecker, J. (2003). Oligomers of the arginine-rich motif of the HIV-1 TAT protein are capable of transferring plasmid DNA into cells. J. Biol. Chem., , 278, 11411-11418.

[98] Sandgren, S, Cheng, F, \& Belting, M. (2002). Nuclear targeting of macromolecular polyanions by an HIV-Tat derived peptide. Role for cell-surface proteoglycans. J. Biol. Chem., , 277, 38877-38883.

[99] Sasaki, K, Kogure, K, Chaki, S, Nakamura, Y, Moriguchi, R, Hamada, H, Danev, R, Nagayama, K, Futaki, S, \& Harashima, H. (2008). An artificial virus-like nano carrier system: enhanced endosomal escape of nanoparticles via synergistic action of $\mathrm{pH}$ sensitive fusogenic peptide derivatives. Analytical and bioanalytical chemistry, , 391, 2717-2727.

[100] Sawant, R. R, Sriraman, S. K, Navarro, G, Biswas, S, Dalvi, R. A, \& Torchilin, V. P. (2012). Polyethyleneimine-lipid conjugate-based $\mathrm{pH}$-sensitive micellar carrier for gene delivery. Biomaterials, , 33(15), 3942-3951.

[101] Scanlon, K. J. (2004). Cancer Gene Therapy: Challenges and Opportunities. Anticancer Research, , 24, 501-504.

[102] Schaffer, D. V, Fidelman, N. A, Dan, N, \& Lauffenburger, D. A. (2000). Vector unpacking as a potential barrier for receptor-mediated polyplex gene delivery. Biotechnology and bioengineering, , 67, 598-606.

[103] Schwarze, S. R, Ho, A, Vocero-akbani, A, \& Dowdy, S. F. (1999). In vivo protein transduction: delivery of a biologically active protein into the mouse. Science, , 285, 1569-1572.

[104] Sethuraman, V. A, Lee, M. C, \& Bae, Y. H. (2008). A biodegradable pH-sensitive micelle system for targeting acidic solid tumors. Pharmaceutical Research, , 25, 657-666.

[105] Shim, M. S, \& Kwon, Y. J. (2012). Stimuli-responsive polymers and nanomaterials for gene delivery and imaging applications. Advanced Drug Delivery Reviews, , 64(11), 1046-1059.

[106] Son, S, Singha, K, \& Kim, W. J. (2010). Bioreducible BPEI-SS-PEG-cNGR polymer as a tumor targeted nonviral gene carrier. Biomaterials, , 31, 6344-6354.

[107] Soudy, R, Gill, A, Sprules, T, Lavasanifar, A, \& Kaur, K. (2011). Proteolytically stable cancer targeting peptides with high affinity for breast cancer cells. Journal of medicinal chemistry, , 54(21), 7523-7534.

[108] Soundara ManickamD. \& Oupický, D. ((2006). Polyplex gene delivery modulated by redox potential gradients. Journal of Drug Targeting, , 14, 519-526.

[109] Stevenson, M, Ramos-perez, V, Singh, S, Soliman, M, Preece, J. A, Briggs, S. S, Read, M. L, \& Seymour, L. W. (2008). Delivery of siRNA mediated by histidine-containing reducible polycations. Journal of Controlled Release, , 130, 46-56. 
[110] Takemoto, H, Ishii, A, Miyata, K, Nakanishi, M, Oba, M, Ishii, T, Yamasaki, Y, Nishiyama, N, \& Kataoka, K. (2010). Polyion complex stability and gene silencing efficiency with a siRNA-grafted polymer delivery system. Biomaterials, , 31, 8097-8105.

[111] Torchilin, V. P, Rammohan, R, Weissig, V, \& Levchenko, T. S. (2001). TAT peptide on the surface of liposomes affords their efficient intracellular delivery even at low temperature and in the presence of metabolic inhibitors. Proc. Natl. Acad. Sci. USA, , 98, 8786-8791.

[112] Torchilin, V. P. (2008). Cell penetrating peptide-modified pharmaceutical nanocarriers for intracellular drug and gene delivery. Peptide Science, , 90, 604-610.

[113] Torchilin, V. P. (2006). Multifunctional nano-carriers. Advanced Drug Delivery Reviews, , 58, 1532-1555.

[114] Trabulo, S, Cardoso, A. L, \& Mano, M. Pedroso de Lima, MC. ((2010). Cell-penetrating peptides-mechanisms of cellular uptake and generation of delivery systems. Pharmaceuticals, , 3, 961-993.

[115] Tung, C. H, Mueller, S, \& Weissleder, R. (2002). Novel branching membrane translocational peptide as gene delivery vector. Bioorg. Med. Chem., , 10, 3609-3614.

[116] Undercover genes slip into the brain(2003). New Scientist, http://www.newscientist.com

[117] Van Vlerken, L. E, Vyas, T. K, \& Amiji, M. M. (2007). Poly (ethylene glycol)-modified nanocarriers for tumor-targeted and intracellular delivery. Pharmaceutical research, , $24,1405-1414$.

[118] Veldhoen, S, Laufer, S. D, \& Restle, T. (2008). Recent developments in peptide-based nucleic acid delivery. Int. J. Mol. Sci., , 9, 1276-1320.

[119] Varkouhi, A. K, Scholte, M, Storm, G, \& Haisma, H. J. (2011). Endosomal escape pathways for delivery of biologicals. Journal of Controlled Release, , 151, 220-228.

[120] Vercauteren, D, Rejman, J, Martens, T. F, Demeester, J, De Smedt, S. C, \& Braeckmans, K. (2012). On the cellular processing of non-viral nanomedicines for nucleic acid delivery: Mechanisms and methods. Journal of Controlled Release, , 161(2), 566-581.

[121] Vidugiriene, J, Goueli, S, \& Sužiedelis, K. (2007). RNA interference: from a research tool to a novel therapeutic agent. Acta Medica Lituanica, , 14(3), 165-169.

[122] Vivès, E, Schmidt, J, \& Pèlegrin, A. (2008). Cell-penetrating and cell-targeting peptides in drug delivery. Biochimica et Biophysica Acta (BBA)-Reviews on Cancer, , 1786, 126-138.

[123] Wadia, J. S, Stan, R. V, \& Dowdy, S. F. (2004). Transducible Tat-HA fusogenic peptide enhances escape of Tat-fusion proteins after lipid raft macropinocytosis. Nat. Med., , 10, 310-315. 
[124] Wagner, E. (2011). Polymers for siRNA delivery: Inspired by viruses to be targeted, dynamic and precise. Accounts of Chemical Research, , 45(7), 1005-1013.

[125] Walker, G. F, Fella, C, Pelisek, J, Fahrmeir, J, Boeckle, S, Ogris, M, \& Wagner, E. (2005). Toward synthetic viruses: endosomal $\mathrm{pH}$-triggered deshielding of targeted polyplexes greatly enhances gene transfer in vitro and in vivo. Molecular Therapy, , 11, 418-425.

[126] Wiethoff, C. M, \& Middaugh, C. R. (2003). Barriers to nonviral gene delivery. Journal of pharmaceutical sciences, , 92, 203-217.

[127] Wu, J, Zhao, X, \& Lee, R. J. (2007). Lipid- based nanoparticulate drug delivery systems. Nano- particulate Drug Delivery Systems. , 89-98.

[128] Xiang, S. D, Scholzen, A, Minigo, G, David, C, Apostolopoulos, V, Mottram, P. L, \& Plebanski, M. (2006). Pathogen recognition and development of particulate vaccines: does size matter? Methods, , 40(1), 1-9.

[129] Yang, L, Yang, H, Rideout, K, Cho, T, Joo, K. I, Ziegler, L, Elliot, A, Walls, A, Yu, D, Baltimore, D, \& Wang, P. (2008). Engineered lentivector targeting of dendritic cells for in vivo immunization. Nat. Biotechnol., , 26(3), 326-334.

[130] Yang, Z. J, Zhang, Y. R, Chen, B, Zhang, S. L, Jia, E. Z, Wang, L. S, Zhu, T. B, Li, C. J, Wang, H, Huang, J, Cao, K. J, Ma, W. Z, Wu, B, Wang, L. S, Wu, C. T, Wang, H, Huang, J, Cao, K. J, Ma, W. Z, Wu, B, Wang, L. S, \& Wu, C. T. (2009). Phase I clinical trial on intracoronary administration of Ad-hHGF treating severe coronary artery disease. Molecular Biology Reports, , 36(6), 1323-1329.

[131] Yu-lin, Y, Wen-teng, C, \& Yuan-wei, S. (2011). Gene therapy using RNAi. Gene Therapy- Developments and Future Perspectives, Intech Open Access

[132] Zatsepin, T. S, Turner, J. J, Oretskaya, T. S, \& Gait, M. J. (2005). Conjugates of oligonucleotides and analogues with cell penetrating peptides as gene silencing agents. Curr. Pharm. Des., , 11, 3639-3654.

[133] Zhang, J, Spring, H, \& Schwab, M. (2001). Neuroblastoma tumor cell-binding peptides identified through random peptide phage display. Cancer letters, , 171, 153-164.

[134] Zhu, L, \& Torchilin, V. R. (2012). Stimulus-responsive nano-preparations for tumor targeting. Integr. Biol. 
